\title{
Phenolic profile, anti-inflammatory, antinociceptive, anti-ulcerogenic and hepatoprotective activities of Pimenta racemosa leaves
}

Fatma Abd-elkader Moharram¹, Amal Amin Al-Gendy ${ }^{2 *}$, Siham Mostafa El-Shenawy ${ }^{3}$, Bassant M. Ibrahim³ and Mohamed A. Zarka ${ }^{4,5}$

\begin{abstract}
Background: Pimenta racemosa tree has many traditional uses where its leaves are used as herbal tea for treatment of flatulence, gastric disorder, osteoarthritis, colds and fever in addition to its analgesic and anti-inflammatory activities. So, this study aimed to isolate phenolic constituents of $80 \%$ aqueous methanol extract (AME) of leaves and evaluate its biological activities.

Methods: The defatted AME was chromatographed and structures of the isolated compounds were elucidated using UV, NMR spectroscopy and UPLC-ESI-MS analysis. Antioxidant activity was investigated using 2, 2-diphenyl-1-picrylhydrazyl (DPPH) radical scavenging capacity. Anti-inflammatory activity was evaluated using carrageenan - induced paw oedema, while antinociceptive activity was determined by chemical and thermal stimuli. Anti-ulcerogenic effect of AME against gastric damage induced by ethanol in Wister male albino rats was evaluated. Also, hepatoprotective activity was investigated through determination of alanine aminotransferase (ALT) and aspartate aminotransferase (AST) following oral administration of paracetamol. Both of Anti-ulcerogenic and hepatoprotective activities (125, 250 and $500 \mathrm{mg} / \mathrm{kg}$ b.wt.) were supported by histopathological examinations.

Results: Gallic acid (1), methyl gallate (2), avicularin (3), quercetin 3-O- $\beta$-D-arbinopyranoside (4), quercetin 3-O- $\beta$-Dglucopyranoside (5), quercetrin (6), cynaroside (7), strictinin (8), castalagin (9), grandinin (10) quercetin (11) and ellagic acid (12) were isolated. AME showed significant radical scavenging activity $\left(\mathrm{SC}_{50}=4.6 \mu \mathrm{g} / \mathrm{mL}\right)$, promising anti-inflammatory effect through inhibition of oedema and antinociceptive activity by reduction in number of writhes after acetic acid injection and prolongation of reaction time towards the thermal stimulus. AME reduced the gastric mucosal lesions compared with ethanol control and ranitidine groups, ALT at the three doses and AST only at 125 and $250 \mathrm{mg} / \mathrm{kg}$ b.wt., when compared with paracetamol group. The results were confirmed by histopathological studies.
\end{abstract}

Conclusion: P. racemosa leaves are rich in phenolic compounds and showed significant biological activities.

Keywords: Anti-inflammatory, Antinociceptive, Anti-ulcerogenic, Hepatoprotective, Myrtaceae, Phenolic compounds, Pimenta racemosa

\footnotetext{
* Correspondence: Amalalgendy@hotmail.com

${ }^{2}$ Department of Pharmacognosy, Faculty of Pharmacy, Zagazig University,

Zagazig 44519, Egypt

Full list of author information is available at the end of the article
}

(c) The Author(s). 2018 Open Access This article is distributed under the terms of the Creative Commons Attribution 4.0 International License (http://creativecommons.org/licenses/by/4.0/), which permits unrestricted use, distribution, and reproduction in any medium, provided you give appropriate credit to the original author(s) and the source, provide a link to the Creative Commons license, and indicate if changes were made. The Creative Commons Public Domain Dedication waiver (http://creativecommons.org/publicdomain/zero/1.0/) applies to the data made available in this article, unless otherwise stated. 


\section{Background}

Pimenta racemosa (Myrtaceae) is commonly known as the West Indian bay tree, bay rum tree and cilimnet. It is native to Carbbean region but it is also cultivated in many warm parts of the world. It is a large tree up to $25 \mathrm{ft}$. high [1]. The traditional uses and pharmacological properties of $P$. racemosa are mostly due to essential oil phenolic constituents, which have shown anti-inflammatory, analgesic, antimicrobial and antioxidant properties [2, 3]. The leaves were indicated for treatment of arthrosis, oedema, gastrosis, inflammation, myalgia and rheumatism in addition to other useful uses [4]. Tea prepared from the leaves of $P$. racemosa was used as a stimulant and for treatment of flatulence, colds and fever. Moreover, the leaf essential oil was used for treatment of both stomach pains, and externally for skin diseases [5] while the bark exhibited strong antischistosomal activity [6]. Antinociceptive and anti-inflammatory effects were also reported for the aqueous extract from leaves of $P$. racemosa var. ozua [7]. Few studies were conducted concerning the previously isolated compounds from Pimenta species. Galloyl glucosides and phenolic glycosides were isolated from $P$. dioica berries $[8,9]$, while galloyl glucosides, flavonoids, tannins and triterpenes were reported in $P$. dioica leaves $[10,11]$. To date, there are no literature reports about the phenolic compounds and pharmacological activities of $P$. racemosa (Mill) J. W. Moore leaves. Inspired by Pimenta dioica leaves which are rich in flavonoids and phenolic compounds exhibited antioxidant and hepatoprotective activities in animal model [12], we were encouraged to investigate $P$. racemosa J.W. Moore leaves for the same effects. Therefore, our objective is to isolate the phenolic constituents from AME of leaves and evaluate its anti-inflammatory, antinociceptive, anti-ulcerogenic and hepatoprotective activities.

\section{Methods}

\section{Plant material}

Leaves of $P$. racemosa (Mill) J. W. Moore (syn. Myrtus caryophyllata, Lacq. Not L, P. acris Kostel), Myrtaceae were collected from Al- Zohria garden, Giza, Egypt during the flowering stage in April 2013. Permission of collection and authentication of the plant was performed by Dr. Trease Labib, former Head of El Orman Botanical Garden, Giza, Egypt which was found matching with the previously reported identification for this plant [6]. Voucher specimen (No.00099CP @ 04-07-04-01) has been deposited in the herbarium of Orman Botanical Garden, Giza, Egypt.

\section{Instruments and materials}

The NMR spectral data were measured using JEOL GX-600 (500 and $100 \mathrm{MHz}$ ) and Bruker (400 and $100 \mathrm{MHz}$ ) for ${ }^{1} \mathrm{H}$ and ${ }^{13} \mathrm{C}$-NMR, respectively. The results were reported as $\delta \mathrm{ppm}$ values relative to TMS as internal reference. UV analyses were measured in $\mathrm{MeOH}$ and different UV shift reagents for pure samples using Shimadzu UV-VIS.1800 spectrophotometer. UV-visible spectrophotometer (Milton Roy, Spectronic 1201) was used for antioxidant activity. For column chromatography, polyamide S (Fluka Chemie AG, Switzerland), sephadex LH-20 (Sigma-Aldrich Steinheim, Germany) and microcrystalline cellulose (E. Merck-Darmstadt, Germany) were used. Whatman No.1 (Whatman Ltd., Maidstone, Kent, England) was used for paper chromatography. Ferric chloride spray reagent was used for visualization of tannins compounds, while Naturstoff reagent and aluminum chloride were used for flavonoids under UV light. $n-\mathrm{BuOH} / \mathrm{HOAc} / \mathrm{H}_{2} \mathrm{O} ; 4: 1: 5 v / \mathrm{v} / \mathrm{v}$, top layer $\left(\mathrm{S}_{1}\right)$ and $15 \%$ aqueous HOAc $\left(\mathrm{S}_{2}\right)$ were used as solvent systems for determination of $R_{f}$ values. Indomethacin, paracetamol and silymarin were obtained from Eipico, Misr and Sedico Companies, Egypt, respectively while ranitidine was obtained from Boehringer Ingelheim $\mathrm{GmbH}$. Kits for alanine aminotransferase (ALT), aspartate aminotransferase (AST) and 2,2-diphenyl-1-picrylhydrazyl (DPPH) obtained from Sigma Chemical Company, USA were used for biological evaluation. All other chemicals and solvent are of high grade.

Compounds 9 and 10 molecular weights were estimated using UPLC-ESI-MS analysis (negative mode) via a UPLC Waters Corporation, Milford MA01757-U.S.A connected to XEVO TQD Triple Quadrupole Mass Spectrometry. Sample $(10 \mu \mathrm{L})$ was injected into ACQUITY UPLC BEH C18 Column (130 A, $1.7 \mu \mathrm{m}, 2.1 \mathrm{~mm} \times 50 \mathrm{~mm})$. A binary gradient of $1 \%$ formic acid in deionized water (solvent $\mathrm{A}$ ) and methanol containing $0.1 \%$ formic acid (solvent $\mathrm{B}$ ) was constructed as follows: $50 \%$ solvent (A): $50 \%$ solvent (B). The flow rate was $1 \mathrm{~mL} / \mathrm{min}$. The mass spectrometer was fitted to an atmospheric pressure electrospray ionization (ESI) source, operated in negative ion mode. The electrospray capillary voltage was set to $3250 \mathrm{~V}$, with a nebulizing gas flow rate of $9 \mathrm{~L} / \mathrm{h}$ and a drying gas temperature of $450{ }^{\circ} \mathrm{C}$. Mass spectrometry data were acquired in the scan mode (mass range $m / z$ 100-1450). The instrument was controlled by Xcalibur software (Xcalibur ${ }^{\mathrm{Tm}} 2.0 .7$, Thermo Scientific).

\section{Extraction and isolation}

Air dried powder of $P$. racemosa leaves was extracted and fractionated according to the procedures of Fig. 1 to afford nine collective fractions (I-IX).

Fraction II was fractionated on cellulose column using water/methanol (10-90\%) mixture to give two sub-fractions, which were further purified on sephadex column using $50 \%$ methanol to give pure sample of 1 and 2. Each of fraction III, VI and VII was chromatographed on cellulose column using BIW (butanol: 


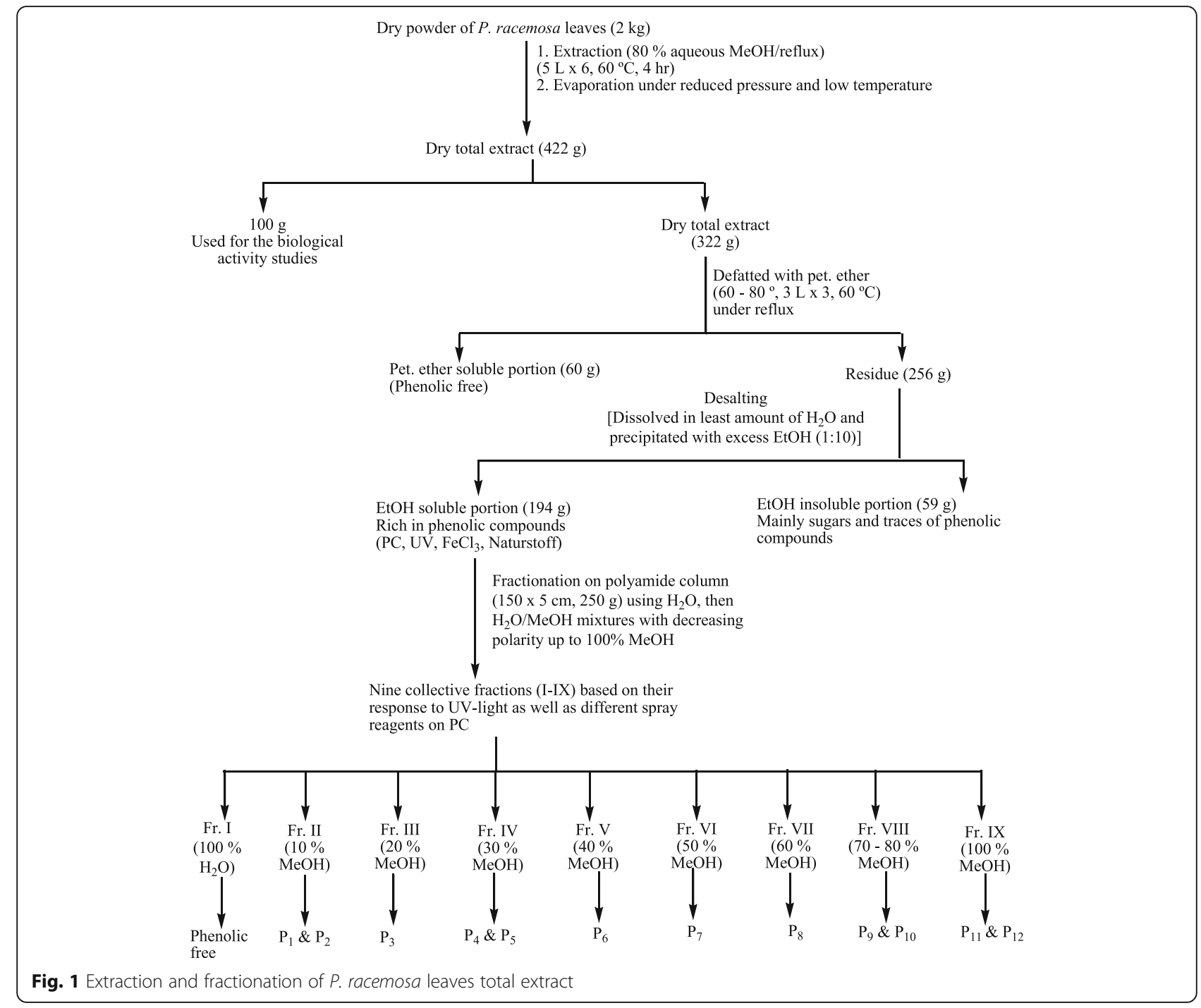

isopropyl alcohol: water, 4:1:5 top layer) followed by sephadex column using methanol/water (1: 1) to give pure samples of 3, 7 and 8 respectively. Fraction IV was purified on cellulose column using water/methanol as an eluent to afford crude samples of 4 and 5 in two sub-fractions. They were further purified using sephadex column using methanol/water (1:1) as eluent to isolate chromatographically pure samples of 4 and 5 . Fraction V was purified using successive columns of cellulose and sephadex (water/methanol mixtures) which led to a pure sample of 6 . Fraction VIII was chromatographed on successive columns of cellulose using ethanol $20-70 \%$ and sephadex with BIW as an eluent to afford crude samples of 9 and 10, which were further purified through precipitation from their ethanolic solutions. Fraction IX was subjected to cellulose column using methanol/water mixture to afford crude samples of 11 and 12, which were purified by precipitation from their solutions.

\section{Biological activities}

\section{DPPH radical scavenging activity}

Freshly prepared $(0.004 \% w / v)$ methanol solution of 2,2-diphenyl-1-picrylhydrazyl (DPPH) radical was used to evaluate antioxidant activity. A $40 \mathrm{uL}$ aliquot of the AME $(2.5-640 \mu \mathrm{g} / \mathrm{mL})$ was added to $3 \mathrm{~mL}$ of DPPH solution and the decrease in absorbance was measured at $515 \mathrm{~nm}$ [13]. The absorbance of the DPPH radical without antioxidant (control) and the reference compound ascorbic acid $(5-80 \mu \mathrm{g} / \mathrm{mL})$ were also measured. All the determinations were performed in three replicates and averaged. The percentage inhibition (PI) of the DPPH radical was calculated according to the formula:

$$
\mathrm{PI}=(\mathrm{AC}-\mathrm{AT}) / \mathrm{AC} \times 100
$$

Where $\mathrm{AC}=$ Absorbance of the control at $t=0 \mathrm{~min}$ and $\mathrm{AT}=$ absorbance of the sample $+\mathrm{DPPH}$ at $t=$ $16 \mathrm{~min}$. 
The percentage of DPPH radical-scavenging was plotted against each applied concentration of AME and ascorbic acid to determine $\mathrm{SC}_{50}$ (the concentration required to scavenge DPPH by $50 \%)$

\section{In vivo studies}

Animals

Wister albino rats of both sexes (150-175 g) were used for acute toxicity while female ones were used for determination of the anti-inflammatory, anti-ulcerogenic and hepatoprotective activities and Swiss mice of both sexes (20-30 g) were used for determination of antinociceptive activity. The animals were obtained from the animal house colony of the National Research Centre, Dokki, Giza, Egypt. The animals were housed in standard metal cages in an air conditioned room at $22 \pm 3^{\circ} \mathrm{C}, 55 \pm 5 \%$ humidity and provided with standard laboratory diet and water ad libitum. Distilled water was used as a vehicle for all extracts and drugs used in the study. All animal procedures were carried out according to the Ethics Committee of the National Research Centre, Cairo, Egypt (Ethics NO. 17-021) and followed the guidelines of the National Institutes of Health Guide for Care and Use of Laboratory Animals.

For anti-ulcerogenic and hepatoprotective experiments, the animals were anesthetized using ether inhalation and euthanized by decapitation.

\section{Acute toxicity study}

The AME was dissolved in distilled water and administered orally to 50 adult Wister albino rats of both sexes (150-175 g) in 5 graded doses up to $5 \mathrm{~g} / \mathrm{kg}$ b.wt., while control group received the same volume of distilled water. Rats were observed for mortality. Experimental doses used were $1 / 40,1 / 20$ and $1 / 10$ of $5 \mathrm{~g} / \mathrm{kg}$ b.wt. of $\operatorname{AME}(125,250$ and $500 \mathrm{mg} / \mathrm{kg}$ b.wt for rats and 175 , 350 and $700 \mathrm{mg} / \mathrm{kg}$ b.wt for mice).

\section{Anti-inflammatory activity}

Paw oedema was induced by sub-plantar injection of right hind paw by $100 \mu \mathrm{l}$ of $1 \%$ sterile lambda carrageenan suspension in saline [14]. The oedema component of inflammation was estimated by measuring hind foot pad immediately before carrageenan injection and 1-4 h post carrageenan injection with a planimeter [15]. Oedema was expressed as a percentage of change from control (pre-drug) values. Forty eight adult Female Wister albino rats (150-175 g) were divided into six groups $(n=8)$. 1st group received orally $1 \mathrm{~mL}$ saline and used as control while 2nd group was sub-plantar injected in right hind paw with $100 \mu \mathrm{L}$ of $1 \%$ carrageenan. 3rd group was given indomethacin $(25 \mathrm{mg} / \mathrm{kg}$ b.wt.) orally as a reference anti-inflammatory drug. The remaining groups received orally 125, 250 and $500 \mathrm{mg} / \mathrm{kg}$. b.wt. of the AME.
All treatments were given orally 60 min before the injection of the carrageenan suspension.

\section{Antinociceptive effect}

It was evaluated by measuring the responses of animals to both a chemical (acetic acid) and a thermal (hot plate) stimulus.

\section{Chemical test}

Induction of writhing in mice using acetic acid was done according to Collier et al. [16]. 30 Swiss mice of both sexes $(20-30 \mathrm{~g})$ were divided into five groups each of six. The 1st control group received saline, while 2nd group received an oral dose of $200 \mathrm{mg} / \mathrm{kg}$ b.wt. of aspirin. 3rd- 5th groups received an oral dose of AME of P. racemosa leaves (125, 250 and $500 \mathrm{mg} / \mathrm{kg}$ b.wt.). After $30 \mathrm{~min}$, the mice of all groups were injected i.p with $0.3 \%$ acetic acid ( $0.2 \mathrm{~mL} /$ mice) and placed in individual cages then the number of writhes was counted and compared in $30 \mathrm{~min}$ period.

\section{Thermal test}

Thermal test was carried out using an electronically controlled hot-plate adjusted to $52{ }^{\circ} \mathrm{C} \pm 0.1{ }^{\circ} \mathrm{C}$ and the cut-off time was 60s [17]. 30 Swiss mice of both sexes (20-30 g) were divided into five groups of mice each of six and they received the same doses as above mentioned in chemical test except the 2nd group was given $20 \mathrm{mg} /$ $\mathrm{kg}$ b.wt. of tramadol orally [18]. The time elapsed until either mice paw licking or jumping occurred was recorded $1 \mathrm{~h}$ before and after 1 and $2 \mathrm{~h}$ post treatment.

\section{Anti-gastroulcerogenic effect}

Gastric lesions were induced in rats by oral administration of $1 \mathrm{~mL} 70 \%$ ethanol single dose [19]. Thirty six adult Female Wister albino rats (150-175 g) were divided into six groups each of six. 1st group received a daily oral dose of $1 \mathrm{~mL}$ distilled water (control group) while 2nd group given orally $1 \mathrm{~mL}$ of $70 \%$ ethanol. $3^{\text {rd }}$ group was orally given $50 \mathrm{mg} / \mathrm{kg}$ b.wt. ranitidine as a reference drug [20] daily for two successive weeks. The remaining groups orally received AME (125, 250 and $500 \mathrm{mg} / \mathrm{kg}$ b.wt.) daily, also for two successive weeks. Ethanol was administered one hour after extract or ranitidine. At the end of the experiment, one hour after the last dose, the rats were sacrificed by cervical dislocation after being lightly anaesthetized. The stomach was excised, opened along the greater curvature, washed with saline and examined thoroughly for mucosal lesions. The number and severity of mucosal lesions were observed and lesions were scaled as follows; petechial lesions $=1$, lesions $<1 \mathrm{~mm}=2$, lesion between 1 and $2 \mathrm{~mm}=3$, lesions between 2 and $4 \mathrm{~mm}=4$, lesions more than $4 \mathrm{~mm}=5$. A total lesion score for each 
animal was calculated by multiplication of the total number of lesions by the respective severity scores. Results are expressed as the severity of lesions per rat [21]. For histopathological study, the stomach was fixed in neutral buffered 10\% formal saline for $72 \mathrm{~h}$, rinsed in tap water and then dehydrated in alcohol (70-95\%), cleared in xylene, impregnated in soft paraffin wax at $55{ }^{\circ} \mathrm{C}$ and finally embedded in hard paraffin. Serial sections of $6 \mu \mathrm{m}$ in thickness were cut and stained with haematoxylin and eosin (H\&E) [22].

\section{Hepatoprotective activity}

Induction of hepatic damage was done by giving paracetamol orally [23]. Thirty six adult Female Wister albino rats $(150-175 \mathrm{~g})$ were divided into six groups each of six. 1st normal control group orally received $1 \mathrm{~mL}$ distilled water while 2 nd group received a single oral dose of paracetamol $(640 \mathrm{mg} / \mathrm{kg}$ b. wt.). 3rd-5th group were given a daily oral dose of $\operatorname{AME}(125,250$ and $500 \mathrm{mg} / \mathrm{kg}$ b. wt., respectively) alone for 15 successive days before paracetamol oral administration and the 6th group was given a daily oral dose of silymarin as a reference drug ( $25 \mathrm{mg} / \mathrm{kg}$ b.wt.) [24] also alone for 15 successive days before paracetamol oral administration. $24 \mathrm{~h}$ after paracetamol administration, the blood was obtained from rato-orbital plexus after anesthesia of rats [25] and centrifuged at $2500 \mathrm{rpm}$ for $15 \mathrm{~min}$. The serum was separated and collected for the determination of ALT and AST. For histopathological study, the liver specimens of all rats were dissected, fixed in $10 \%$ neutral-buffered normal saline for $72 \mathrm{~h}$, washed with water and then dehydrated in grades of alcohol (70-95\%), cleared in xylene and embedded in paraffin. Serial sections of $6 \mu \mathrm{m}$ thick were cut and stained with haematoxylin and eosin [22].

\section{Statistical analysis}

Values were expressed as means \pm S.E. Comparisons between means were carried out using one way analysis of variance (ANOVA) followed by Tukey Kramer multiple comparisons test for all tests except for the thermal test which was conducted using hot plate and the anti-inflammatory test where comparisons between means were carried out using two way ANOVA followed by Bonferroni's multiple comparisons test. $P \leq 0.05$ was accepted as being significant in all types of statistical tests. Graph pad prism software (version 6) was used to carry out all statistical tests.

\section{Results}

\section{Chromatographic and spectroscopic data of the isolated compounds}

Two dimensional paper chromatography (2D-PC) screening of $P$. racemosa leaves' AME revealed the presence of a mixture of phenolic compounds based on their color properties under UV-light and their response to different spray reagents. Fractionation of AME on a polyamide column, followed by purification on cellulose and Sephadex LH-20 columns resulted in the isolation of 12 compounds. Five flavonols, one flavone, three tannins in addition to two phenolic acids and one of their derivatives were isolated and identified. All compounds were isolated from P. racemosa leaves for the first time while compounds 3-7 were isolated from genus Pimenta for the first time. The structures of these compounds (Fig. 2) were fully elucidated on the basis of their physicochemical and spectral data (UV, ${ }^{1} \mathrm{H}-\mathrm{NMR},{ }^{13} \mathrm{C}$-NMR and UPLC-ESI-MS) and by comparison with published data and authentic samples.

Both of compounds 1 (45 mg) and $2(27 \mathrm{mg})$ were obtained as off-white amorphous powder. They gave shine violet fluorescent spots under short UV- light and blue color with $\mathrm{Fe} \mathrm{Cl}_{3}$ spray reagent. $\mathrm{R}_{\mathrm{f}}$ values are 0.14 $\left(\mathbf{S}_{\mathbf{1}}\right)$ and $0.75\left(\mathbf{S}_{\mathbf{2}}\right)$ for $\mathbf{1}$ and $0.18\left(\mathbf{S}_{\mathbf{1}}\right)$ and $0.69\left(\mathbf{S}_{\mathbf{2}}\right)$ for $\mathbf{2}$. The identification was based upon Co-PC with available authentics and comparison of spectroscopic data with the matched literature as will be discussed later.

Compounds 3 (22 mg), 4 (17 mg), $5(20 \mathrm{mg})$ and 6 $(25 \mathrm{mg})$ were purified as yellow amorphous powder showing deep purple fluorescence under long UV. They gave green and orange colour when sprayed by ferric chloride and Naturstoff reagents, respectively. Their $R_{f}$ values, UV, ${ }^{1} \mathrm{H}$ and ${ }^{13} \mathrm{C}$-NMR data are listed in Tables 1 and 2.

Compound 7: Yellow amorphous powder $(20 \mathrm{mg})$ with $R_{\mathrm{f}}$ values $0.42\left(\mathbf{S}_{\mathbf{1}}\right)$ and $0.3\left(\mathbf{S}_{\mathbf{2}}\right)$ and giving dark purple spot under long UV that changed to orange fluorescence and pale green color with Naturstoff and $\mathrm{FeCl}_{3}$ spray reagents, respectively. UV spectral data $\lambda_{\max }(\mathrm{nm}) \mathrm{MeOH}: 255,269$ (sh), 345; (+NaOMe): 258300 (sh), 400; (+NaOAc): 254 (sh), 268, 358 (sh), 390; (+NaOAc $\left./ \mathrm{H}_{3} \mathrm{BO}_{3}\right): 260,294$ (sh), $365\left(+\mathrm{AlCl}_{3}\right): 265,300$ (sh), 430; $\left(+\mathrm{AlCl}_{3} / \mathrm{HCl}\right): 270,283$ (sh), 365. ${ }^{1} \mathrm{H}-\mathrm{NMR}$ $\left(400 \mathrm{MHz}, \mathrm{DMSO}, d_{6}\right) \delta \mathrm{ppm} 13.00(1 \mathrm{H}, s, \mathrm{H}$-bonded $\mathrm{OH}-5), 7.45\left(2 \mathrm{H}, m \mathrm{H} 2^{\prime} / 6^{\prime}\right), 6.90\left(1 \mathrm{H}, d, \mathrm{~J}=8.0, \mathrm{H}-5^{\prime}\right)$, $6.79(1 \mathrm{H}, s, \mathrm{H}-3), 6.77(1 \mathrm{H}, d, \mathrm{~J}=1.2, \mathrm{H}-8), 6.45(1 \mathrm{H}, d$, $\mathrm{J}=1.2, \mathrm{H}-6), 5.09(1 \mathrm{H}, d, \mathrm{~J}=6.8 \mathrm{~Hz}, \mathrm{H}-1 ")$ and $3.72-3.24$ (m, remaining sugar protons). ${ }^{13} \mathrm{C}-\mathrm{NMR}(100 \mathrm{MHz}$, DMSO, $\left.d_{6}\right) 182.36$ (C-4), 164.94 (C-2), 163.42 (C-7), 161.60 (C-5), 157.41 (C-9), 150.39 (C-4'), 146.25 (C-3'), 121.85 (C-1'), 119.63 (C-6'), 116.45 (C-2'), $114.04\left(\mathrm{C}-5^{\prime}\right)$, 105.80 (C-10), 103.64 (C-3), 100.36 (C-6), 100.00 (C-1"), 95.19 (C-8), 77.63 (C-5”), 76.86 (C-3"), 73.58 (C-2”), $70.02(\mathrm{C}-4 ")$ and 61.08 (C-6").

Compound 8: Brown amorphous powder (15 mg) with $R_{\mathrm{f}}$ values $0.27\left(\mathbf{S}_{\mathbf{1}}\right)$ and $0.36\left(\mathbf{S}_{\mathbf{2}}\right)$; It gave dark purple fluorescent spot under short UV-light, turned to indigo-red and deep blue color with $\mathrm{NaNO}_{2}$-glacial $\mathrm{AcOH}$ and $\mathrm{FeCl}_{3}$ spray reagents, respectively. UV $\lambda_{\max }(\mathrm{nm})$; 
<smiles>COC(=O)c1cc(O)c(O)c(O)c1</smiles>

$$
\text { 1: } \mathrm{R}=\mathrm{H}
$$$$
\text { 2: } \mathrm{R}=\mathrm{CH}_{3}
$$

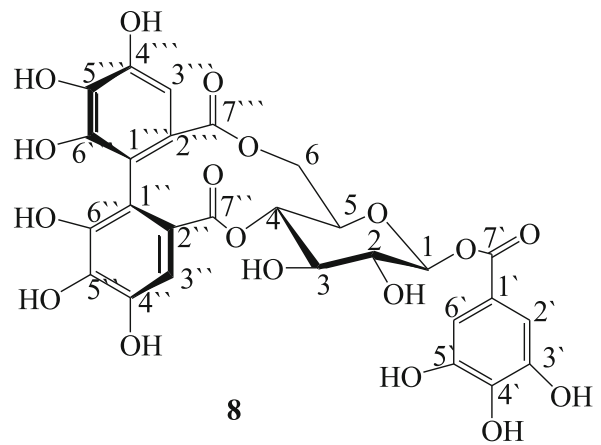<smiles>O=c1oc2c(O)c(O)cc3c(=O)oc4c(O)c(O)cc1c4c23</smiles><smiles>[R20]c1cc(O)c2c(=O)c([R])c(-c3ccc(O)c(O)c3)oc2c1</smiles>

$3: \mathrm{R}_{1}=O$-L-arabinofuranoside; $\mathrm{R}_{2}=\mathrm{H}$

4: $\mathrm{R}_{1}=O$-D-arbinopyranoside; $\mathrm{R}_{2}=\mathrm{H}$

5 : $\mathrm{R}_{1}=O$-D-glucopyranoside; $\mathrm{R}_{2}=\mathrm{H}$

$6: \mathrm{R}_{1}=O$-L-rhamnopyranoside; $\mathrm{R}_{2}=\mathrm{H}$

$7: \mathrm{R}_{1}=\mathrm{H} ; \mathrm{R}_{2}=O$-D-glucopyranoside

11: $\mathrm{R}_{1}=\mathrm{OH} ; \mathrm{R}_{2}=\mathrm{H}$

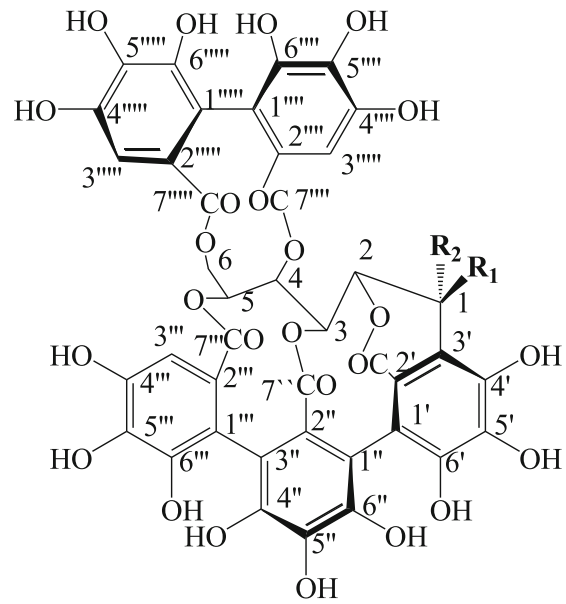

$9: \mathrm{R}_{1}=\mathrm{H} ; \mathrm{R}_{2}=\mathrm{OH}$

$10: \mathrm{R}_{1}=\mathrm{H} ; \mathrm{R}_{2}=$ Pentose

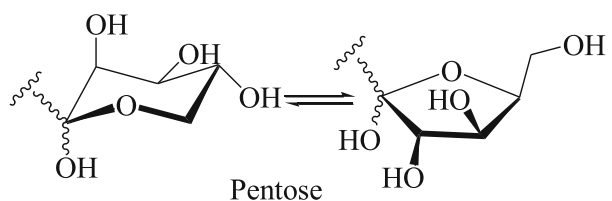

Fig. 2 Structure of the compounds isolated from $P$. racemosa leaves AME

Table $1 R_{f}$ values and UV data of compounds 3-6

\begin{tabular}{|c|c|c|c|c|c|c|c|c|}
\hline \multirow[t]{2}{*}{ Compound } & \multicolumn{2}{|c|}{$R_{f}$ values } & \multicolumn{6}{|l|}{ UV data $\lambda_{\max }(\mathrm{nm})$} \\
\hline & $\mathrm{S}_{1}$ & $\mathrm{~S}_{2}$ & $\mathrm{MeOH}$ & $+\mathrm{NaOMe}$ & $+\mathrm{NaOAC}$ & $+\mathrm{NaOAC}+\mathrm{H}_{3} \mathrm{BO}_{3}$ & $+\mathrm{AlCl}_{3}$ & $+\mathrm{AlCl}_{3}+\mathrm{HCl}$ \\
\hline 3 & 0.5 & 0.52 & $\begin{array}{l}256,295 \text { (sh), } \\
352\end{array}$ & $\begin{array}{l}269,325 \text { (sh), } \\
403\end{array}$ & $\begin{array}{l}270,320 \text { (sh), } \\
367\end{array}$ & $269,310,402$ & $\begin{array}{l}270,306 \text { (sh), } 335 \text { (sh), } \\
419\end{array}$ & $\begin{array}{l}267,293 \text { (sh), } 358 \text { (sh) } \\
395\end{array}$ \\
\hline 4 & 0.47 & 0.54 & 254, 290 (sh), 354 & 270,329 (sh), 410 & 271,317 (sh), 372 & $270,299,405$ & 273,320 (sh), 410 & $272,290,365$ (sh), 399 \\
\hline 5 & 0.41 & 0.6 & 255, 264 (sh), 358 & 273,409 & 270,375 & 268, 293 (sh), 375 & 259 (sh), 265, 271, 409 & 252 (sh), 269, 365 \\
\hline 6 & 0.43 & 0.55 & 252, 299 (sh), 355 & 265,322 (sh), 415 & 269,320 (sh), 368 & 264, 305 (sh), 377 & 272,302 (sh), 315, 420 & 269, 305 (sh), 399 \\
\hline
\end{tabular}


Table $2{ }^{1} \mathrm{H}$ and ${ }^{13} \mathrm{C}-\mathrm{NMR}$ data of compounds 3-6

\begin{tabular}{|c|c|c|c|c|c|c|c|c|}
\hline \multirow[t]{3}{*}{ C No. } & \multicolumn{2}{|l|}{3} & \multicolumn{2}{|l|}{4} & \multicolumn{2}{|l|}{5} & \multicolumn{2}{|l|}{6} \\
\hline & \multicolumn{2}{|c|}{3 DMSO-d6, 500 and $125 \mathrm{MHz}$} & \multicolumn{2}{|c|}{$4\left(\mathrm{CD}_{3} \mathrm{OD}-d 4,400\right.$ and $\left.100 \mathrm{MHz}\right)$} & \multicolumn{2}{|c|}{5 DMSO-d6, 400 and $100 \mathrm{MHz}$} & \multicolumn{2}{|c|}{$6 \mathrm{DMSO}-\mathrm{d} 6,400$ and $100 \mathrm{MHz}$} \\
\hline & $\delta_{H}$ & $\delta_{c}$ & $\delta_{H}$ & $\delta_{c}$ & $\delta_{H}$ & $\delta_{c}$ & $\delta_{H}$ & $\delta_{c}$ \\
\hline 2 & & 157.49 & & 157.39 & & 157.00 & & 157.61 \\
\hline 3 & & 133.85 & & 134.21 & & 133.58 & & 134.57 \\
\hline 4 & & 178.19 & & 178.10 & & 177.48 & & 178.07 \\
\hline 5 & & 161.72 & & 161.59 & & 161.56 & & 161.73 \\
\hline 6 & \multirow[t]{2}{*}{$6.16 d(1.9)$} & 99.19 & \multirow[t]{2}{*}{6.23 br s } & 98.57 & \multirow[t]{2}{*}{$6.11 b r s$} & 99.83 & \multirow[t]{2}{*}{$6.17 \mathrm{brs}$} & 99.36 \\
\hline 7 & & 164.83 & & 164.62 & & 167.27 & & 165.50 \\
\hline 8 & \multirow[t]{4}{*}{$6.37 d(1.9)$} & 94.08 & \multirow[t]{4}{*}{6.43 br s } & 93.39 & \multirow[t]{7}{*}{6.31 br s } & 94.34 & \multirow[t]{4}{*}{6.35 br s } & 94.18 \\
\hline 9 & & 156.86 & & 157.00 & & 156.24 & & 156.97 \\
\hline 10 & & 104.42 & & 104.23 & & 103.44 & & 104.23 \\
\hline $1^{\prime}$ & & 122.24 & & 121.71 & & 121.96 & & 121.54 \\
\hline $2^{\prime}$ & \multirow[t]{3}{*}{$7.44 d(1.95)$} & 116.04 & \multirow[t]{3}{*}{$7.76 d(1.6)$} & 114.85 & & 115.72 & \multirow[t]{3}{*}{7.29 br s } & 115.91 \\
\hline $3^{\prime}$ & & 145.59 & & 144.58 & & 145.46 & & 145.71 \\
\hline $4^{\prime}$ & & 148.99 & & 148.57 & & 149.34 & & 148.99 \\
\hline $5^{\prime}$ & $6.82 d(8.3)$ & 115.98 & $6.90 d(8.4)$ & 116.09 & \multirow[t]{2}{*}{$6.83 d(8.4)$} & 116.51 & $6.88 d(8.4)$ & 115.72 \\
\hline $6^{\prime}$ & \multirow[t]{2}{*}{$7.51 d d(1.95,8.3)$} & 121.43 & $7.59 d d(1.6,8.4)$ & 121.44 & & 121.39 & $7.25 d d(b r s, 8.4)$ & 121.13 \\
\hline $2^{\prime} / 6^{\prime}$ & & & & & \multicolumn{2}{|l|}{$7.58 \mathrm{~m}$} & & \\
\hline$H-1^{\prime \prime}$ & $5.55 b r s$ & 108.29 & $5.14 d(6.0)$ & 103.22 & $5.44 d(6.8)$ & 101.57 & 5.25 br s & 102.25 \\
\hline$H-2^{\prime \prime}$ & $4.11 \mathrm{~m}$ & 82.63 & \multirow{5}{*}{$\begin{array}{l}3.94-3.46 \mathrm{~m} \text { remaining } \\
\text { sugar protons }\end{array}$} & 72.71 & \multirow{5}{*}{$\begin{array}{l}\text { 3.6-3.17 } m \text {, remaining } \\
\text { sugar protons }\end{array}$} & 74.58 & $3.53 d d(3.2,2.0) 3.4-$ & 70.79 \\
\hline H-3" & \multirow{4}{*}{$\begin{array}{l}3.49-3.29 \mathrm{~m} \text {, remaining } \\
\text { sugar protons }\end{array}$} & 77.39 & & 71.46 & & 76.98 & \multirow{3}{*}{$\begin{array}{l}3.1 \mathrm{~m} \text {, remaining } \\
\text { sugar protons }\end{array}$} & 71.62 \\
\hline$H-4^{\prime \prime}$ & & 86.28 & & 67.75 & & 70.36 & & 71.04 \\
\hline$H-5^{\prime \prime}$ & & 62.56 & & 65.63 & & 77.93 & & 70.51 \\
\hline$H-6^{\prime \prime}$ & & & & & & 61.40 & $1.09 d(6.1)$ & 17.96 \\
\hline
\end{tabular}

$\mathrm{J}$-values $(\mathrm{Hz})$ were reported in parenthesis

MeOH: 246, 288 sh. ${ }^{1} \mathrm{H}-\mathrm{NMR}$ (400 MHz, DMSO- $d_{6}: \delta$ ppm $6.97\left(2 \mathrm{H}, \mathrm{s}, \mathrm{H}-2^{\prime} / 6\right.$ ' galloyl moiety), $6.42(1 \mathrm{H}, s$, H-3"'HHDP), $6.40(1 \mathrm{H}, s, \mathrm{H}-3$ " HHDP), $5.70(1 \mathrm{H}, d, \mathrm{~J}=$ $8.4 \mathrm{~Hz}, \mathrm{H}-1), 4.86\left(1 \mathrm{H}, d d, \mathrm{~J}=12.8,5.2 . \mathrm{Hz}, \mathrm{H}-6_{\mathrm{a}}\right), 4.60$ $(1 \mathrm{H}, \mathrm{t}$-like, $\mathrm{J}=10 \mathrm{~Hz}, \mathrm{H}-4), 4.40(1 \mathrm{H}, b r d d, \mathrm{~J}=12.8$, $9.2 \mathrm{~Hz}, \mathrm{H}-5), 3.68\left(1 \mathrm{H}, b r d, \mathrm{~J}=14.0 \mathrm{~Hz}, \mathrm{H}-6_{\mathrm{b}}\right), 3.63(1 \mathrm{H}$, t-like, $\mathrm{J}=7.6 \mathrm{~Hz}, \mathrm{H}-3)$ and $3.26(1 \mathrm{H}, \mathrm{t}$-like, $\mathrm{J}=10.0 \mathrm{~Hz}$, H-2). ${ }^{13} \mathrm{C}-\mathrm{NMR}\left(100 \mathrm{MHz}, \mathrm{DMSO}-d_{6}\right): \delta \mathrm{ppm} 167.83$, 167.09 (C-7"'/7"HHDP), 164.62 (C-7' G), 145.62 (C-3'/5' G), 144.50, 144.41 (C-6"/6" HHDP), 144.26. 144.23 (C-4"/ 4"' HHDP), 139.17 (C-4' G), 135.33, 134.96 (C-5"/5"' HHDP), 124.70, 124.42 (C-2"/2"' HHDP), 118.42 (C-1' G), 115.55, 115.23 (1"/1"' HHDP), 109.09 (C-2'/6' G), 106.29, 105.51 (C-3"/ 3"' HHDP), 94.73 (C-1), 73.82 (C-3), 73.33 (C-5), $71.61(\mathrm{C}-4 / 2)$ and $62.74(\mathrm{C}-6)$.

Compound 9: It was obtained as brown amorphous powder $(25 \mathrm{mg})$ with $\mathrm{R}_{\mathrm{f}}$ value $0.59\left(\mathrm{~S}_{2}\right)$. It also showed dark purple fluorescent spot under short UV-light and dull brown under long UV-light, turned to indigo-red with $\mathrm{NaNO}_{2}$ - glacial $\mathrm{AcOH}$ and deep blue color with $\mathrm{FeCl}_{3}$, respectively. UV $\lambda_{\max }(\mathrm{nm}) ; \mathrm{MeOH}: 248,290$ (sh). ${ }^{1} \mathrm{H}-\mathrm{NMR}$ (400 $\left.\mathrm{MHz}, \quad \mathrm{CD}_{3} \mathrm{OD}-d_{4}\right), \delta$ ppm $6.69(2 \mathrm{H}, s, \quad \mathrm{H}-3$ ”' flavogalonic acid (FLG); H-3"' HHDP), 6.50 (1 H, $s, \mathrm{H}-3^{\text {"'”' }}$ HHDP), $5.51(1 \mathrm{H}, d, \mathrm{~J}=4.0 \mathrm{~Hz}, \mathrm{H}-1), 5.48(1 \mathrm{H}, d, \mathrm{~J}=$ $8.0 \mathrm{~Hz}, \mathrm{H}-5), 5.07(1 \mathrm{H}, \mathrm{t}-\mathrm{like}, \mathrm{J}=8.0 \mathrm{~Hz}, \mathrm{H}-4), 4.93(1 \mathrm{H}, d d$, $\mathrm{J}=4.0,12.0 \mathrm{~Hz}, \mathrm{H}-6), 4.82(2 \mathrm{H}, m, \mathrm{H}-3 / 2$ hidden by water signal) and $3.88\left(1 \mathrm{H}, d, \mathrm{~J}=12.0 \mathrm{~Hz}, \mathrm{H}-6{ }^{\prime}\right) .{ }^{13} \mathrm{C}-\mathrm{NMR}$ $\left(100 \mathrm{MHz}, \mathrm{CD}_{3} \mathrm{OD}-d_{4}\right): \delta \mathrm{ppm} 169.01,166.87$ (C-7"'/C-7"'”' HHDP), 166.15 (C-7"' FLG), 165.01 (C-7” FLG), 164.96 (C-7' FLG), 146.08 (C-4"' FLG), 144.79, 144.68 (C-6"'"/6"'” HHDP), 144.66, 143.92 (C-4'"'/4"'” HHDP, 4'/4" FLG), 143.58, 142.27, 143.05 (C-6'/6"/6"' FLG), 136.96 (C-5" FLG), 136.51, 136.40 (C-5"'/5"'” HHDP), 135.87 (C-5" FLG), 134.65 (C-5”' FLG), 126.63 (C-2” FLG), 125.43, 123.99 (C-2"'/2"'” HHDP), 123.83 (C-2"' FLG), 120.68 (C-2'FLG), 116.02, 115.28, 114.99, 114.45, 113.85, 113.78 (C-1"'/1"'” HHDP, C-1'/1"/1"' FLG, C-3' FLG), 112.33 (C-3" FLG), 108.22, 107.70, 106.93 (C-3"' FLG, C-3"'"/3"'” HHDP), 73.68 (C-2), 70.77 (C-5), 68.63 (C-4), 66.35 (C-1), $65.55(\mathrm{C}-3)$ and 64.64 (C-6).

UPLC-ESI-MS (negative mode), $m / z$ (relative abundance \%): 933 ([M-H] $\left.]^{-}, 100 \%\right)$.

Compound 10: It was purified as brown amorphous powder (30 mg) with $R_{f}$ value $0.61\left(S_{2}\right)$. It also gave dark 
purple fluorescent spot under short UV-light and dull brown under long UV-light, turned to indigo-red with $\mathrm{NaNO}_{2}$ - glacial AcOH and deep blue color with $\mathrm{FeCl}_{3}$, respectively. UV $\lambda_{\max }(\mathrm{nm}) ; \mathrm{MeOH}: 245,293$ (sh). ${ }^{1} \mathrm{H}-\mathrm{NMR}\left(400 \mathrm{MHz}, \mathrm{CD}_{3} \mathrm{OD}, d_{4}\right): \delta \mathrm{ppm} 7.32,6.79,6.79$, 6.79, 6.78 (1H in total, each s, H-6"'), 6.63, 6.61, 6.62, 6.59 (2H in total, each s, H-6"'/6"'”), 5.78, 5.63, 5.61, 5.52, 5.23, $5.21,5.20,5.18,5.16,5.14(2 \mathrm{H}$ in total, $m, \mathrm{H}-2 / 5)$, 4.87( $\mathrm{H}-4$ hidden by $\mathrm{H}_{2} \mathrm{O}$ signal), $4.53(1 \mathrm{H}, d d, \mathrm{~J}=8.0$, $\left.16.0, \mathrm{~Hz}, \mathrm{H}-6_{\mathrm{a}}\right), 4.28(1 \mathrm{H}, b r d, \mathrm{~J}=8 \mathrm{~Hz}, \mathrm{H}-3), 4.01,3.98$ $(1 \mathrm{H}$, in total, each $b r s, \mathrm{H}-1), 3.90-3.32\left(4 \mathrm{H}, \mathrm{m}, \mathrm{H}-6_{\mathrm{b}}, \mathrm{H}-4\right.$ pentoses, $2 \mathrm{H}-5$ pentoses $) .{ }^{13} \mathrm{C}-\mathrm{NMR}\left(100 \mathrm{MHz}, \mathrm{CD}_{3} \mathrm{OD}-d_{4}\right)$ : $\delta$ ppm 169.23, 169.04 (C-7"'”), 166.77, 166.53 (C-7"'), 166.37 (C-7"'), 165.78 (C-7"), 165.78, 164.72, 164.74 (C-7'), 148.14, 146.73, 146.33, (C-5"'), 144.78, 144.74 (C-3"', 3"'”), 144.69 (2C, C-5"'”, 5'”'), 144.31, 143.89, 144.81, 143.57, 143.49 (C-5", 5'), 143.39, 143.37, 143.31, 142.64 (C-3', 3", 3"'), 137.73, 137.28, 137.18 (C-4"'), 136.89, 136.61, 136.39, 136.29, 135.80 (C-4"', 4'”'), 135.62, 134.70 (C-4"), 134.67 (C- 4'), 126.66, 126.50, 126.39 (C-1"), 125.63, 125.49, 125.44, 125.24 (C-1"', 1'"')), 123.89, 123.84 (C-1"), 123.42 (C-1'), 115.72, 115.40, 114.90, 114.58, 114.41, 114.18, 114.12, 113.87, 113.84, 113.03 (C-2', 2", 2', 2"', 2'"'), 112.26 (C-6'), 112.14, 110.40, 109.21 (C-6"), 108.04, 107.80, (C-6"'), 106.78, 106.53, (C-6"', 6"'”). Sugar moieties: 101.15,101.07, 99.42, 99.34 (C-1 pentose), 80.08, 79.3, 72.09, 69.10 (C-4 pentose), 74.87, 74.38 (C-2 pentose), 73.46 (C-3 pentose), 71.87, 71.50 (C-2), 71.43, 71.38 (C-5), $70.64,70.47,70.29,69.96$ (C-4), 69.49, 69.00, 68.97, 68.38

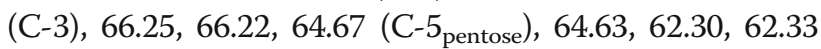
(C-6), 47.03, 46.96 and 45.86 (C-1).

UPLC-ESI-MS (negative mode), $\mathrm{m} / z$ (relative abundance \%): $1065\left([\mathrm{M}-\mathrm{H}]^{-}, 100 \%\right)$.

Compound 11: It was obtained as yellow amorphous powder $\left(18 \mathrm{mg}\right.$ ) with $\mathrm{R}_{\mathrm{f}}$ value $0.75\left(\mathbf{S}_{\mathbf{1}}\right)$ and giving dark purple spot under long UV that changed to orange fluorescence and pale green color with Naturstoff and $\mathrm{FeCl}_{3}$ spray reagents, respectively.

Compound 12: It was obtained as off white amorphous powder $\left(15 \mathrm{mg}\right.$ ) with $\mathrm{R}_{\mathrm{f}}$ value $0.25\left(\mathbf{S}_{\mathbf{1}}\right)$. It gave shine violet fluorescent spots under short UV- light and blue color with $\mathrm{Fe} \mathrm{Cl}_{3}$ spray reagent. The identification was based upon Co-PC with available authentics and comparison of spectroscopic data with the available literature as will be mentioned later.

\section{Biological activities}

The DPPH scavenging percentages of AME and ascorbic acid as well as $\mathrm{SC}_{50}$ values indicated their significant antioxidant activities. AME showed promising antioxidant activities as indicated by its high DPPH scavenging percentage $(89.67 \%)$ at $320 \mu \mathrm{g} / \mathrm{mL}$ and low $\mathrm{SC}_{50}$ value $(4.6 \mu \mathrm{g} / \mathrm{mL})$ when compared with the activity of the standard ascorbic acid (92.48\% scavenging percentage at $40 \mu \mathrm{g} / \mathrm{mL}$ and $\mathrm{SC}_{50}=14.2 \mu \mathrm{g} / \mathrm{mL}$ ).

Acute toxicity study revealed that AME of $P$. racemosa is non-toxic up to $5 \mathrm{~g} / \mathrm{kg}$ b.wt. After $24 \mathrm{~h}$, there is no mortality recorded, and as Semler et al. reported [26], if just one dose level at $5 \mathrm{~g} / \mathrm{kg}$ b.wt. is not lethal to animals, there is no need to determine $\mathrm{LD}_{50}$.

Concerning the anti-inflammatory activity, oral administration of $125 \mathrm{mg} / \mathrm{kg}$ b.wt. AME exhibited significant inhibition of oedema formation by 42.1, 45.01 and $26.68 \%$ after 2 nd, 3rd and 4th h, respectively after carrageenan injection as compared with saline group at the same time post carrageenan injection, while 250 and $500 \mathrm{mg} / \mathrm{kg}$ b.wt. induced significant inhibition of oedema formation by $23.34,75.66,76.87$ and $50.66 \%$ for $250 \mathrm{mg} / \mathrm{kg}$ b.wt. and $61.46,80.53,86.12$ and $93.83 \%$ for $500 \mathrm{mg} / \mathrm{kg}$ b.wt. after $1 \mathrm{st}$, 2nd, 3rd and $4^{\text {th }} \mathrm{hr}$, respectively post carrageenan injection in comparison to saline group. Moreover, administration of $500 \mathrm{mg} / \mathrm{kg}$ b.wt. of AME exhibited significant inhibition of oedema which exceeded the activity of indomethacin after 1st, 2nd, 3rd and 4 th $h$ that was indicated by the percentage of inhibition values (Table 3 ).

Regarding the antinociceptive activity either by chemical (acetic acid test) or thermal (hot-plate test) stimuli, it was found that AME caused significant decrease in the number of writhes in mice after acetic acid injection in dose-dependent manner being 18.66, 40.00 and $49.33 \%$ at 175,350 and $700 \mathrm{mg} / \mathrm{kg}$ b.wt., respectively upon comparison with saline control (Table 4). AME $(700 \mathrm{mg} / \mathrm{kg}$ b.wt.) showed a significant prolongation of the reaction time to the thermal stimulus by 23.28 and $45.15 \%$ after 1 and $2 \mathrm{~h}$, respectively as compared with control pre-drug (Table 5).

Concerning gastric ulcerogenic effect both the number and severity of gastric mucosal lesions induced by ethanol were reduced after oral administration of AME (125, 250 and $500 \mathrm{mg} / \mathrm{kg}$ ) by $46.9 \& 52.8 \%, 79 \& 82.4$ and $82.7 \%$ \& $91.5 \%$, respectively relative to the control values while ranitidine showed reduction in the number and severity by $87.7 \& 94.4 \%$, respectively by comparison with ethanol groups (Table 6).

The histopathological examination of gastric mucosa showed normal gastric mucosa in the control group which was treated with $1 \mathrm{~mL}$ saline (Fig. $3 \mathrm{a} \& \mathrm{~b}$ ) while oral ethanol administration induced necrosis of apical mucosa accompanied by hemorrhage and submucosal edema (Fig. $3 \mathrm{c}$ and d). Oral administration of AME in three doses induced gastro-protective effect which confirmed the results of naked eye evaluation of ulcer number and severity (Fig. $3 \mathrm{f}$ and k). Administration of the AME exhibited a significant hepatoprotective effect in a dose dependent manner in paracetamol induced hepatotoxicity rats. Oral administration of a single dose 
Table 3 Anti-inflammatory activity of $P$. racemosa leaves AME and indomethacin on carrageenan-induced paw oedema in rats

\begin{tabular}{|c|c|c|c|c|c|c|c|c|c|}
\hline \multicolumn{10}{|c|}{ Oedema } \\
\hline $\begin{array}{l}\text { Drug (mg/kg } \\
\text { b.wt. P.O) }\end{array}$ & $\begin{array}{l}\text { Zero time } \\
(\mathrm{cm})\end{array}$ & $\begin{array}{l}1 \mathrm{~h}(\mathrm{~cm}) \\
\text { (\% increase) }\end{array}$ & $\begin{array}{l}\% \text { of } \\
\text { Inhibition }\end{array}$ & $\begin{array}{l}2 \mathrm{~h} \text { (cm) } \\
(\% \text { increase) }\end{array}$ & $\begin{array}{l}\% \text { of } \\
\text { Inhibition }\end{array}$ & $\begin{array}{l}3 \mathrm{~h}(\mathrm{~cm}) \\
\text { (\% increase) }\end{array}$ & $\begin{array}{l}\% \text { of } \\
\text { Inhibition }\end{array}$ & $\begin{array}{l}4 \mathrm{~h}(\mathrm{~cm}) \\
\text { (\% increase) }\end{array}$ & $\begin{array}{l}\% \text { of } \\
\text { Inhibition }\end{array}$ \\
\hline \multirow[t]{2}{*}{ Saline } & \multirow[t]{2}{*}{$0.37 \pm 0.01$} & $0.49 \pm 0.01$ & \multirow[t]{2}{*}{-} & $0.56 \pm 0.03$ & \multirow[t]{2}{*}{-} & $0.57 \pm 0.009$ & \multirow[t]{2}{*}{-} & $0.52 \pm 0.02$ & \multirow[t]{2}{*}{-} \\
\hline & & $(32.43 \pm 0.3)$ & & $(51.35 \pm 0.54)^{*}$ & & $(54.05 \pm 0.63)^{*}$ & & $(40.54 \pm 0.6)$ & \\
\hline Indomethain & \multirow[t]{2}{*}{$0.36 \pm 0.01$} & $0.48 \pm 0.02$ & \multirow[t]{2}{*}{2.68} & $0.45 \pm 0.02$ & \multirow[t]{2}{*}{51.31} & $0.44 \pm 0.006$ & \multirow[t]{2}{*}{58.88} & $0.4 \pm 0.01$ & \multirow[t]{2}{*}{72.59} \\
\hline (25) & & $(33.3 \pm 0.21)^{*}$ & & $(25 \pm 0.25)^{*}$ & & $(22.22 \pm 0.22)^{*}$ & & $(11.11 \pm 0.38)^{*}$ & \\
\hline \multicolumn{10}{|l|}{ AME } \\
\hline \multirow[t]{2}{*}{ (125) } & \multirow[t]{2}{*}{$0.37 \pm 0.01$} & $0.47 \pm 0.007$ & \multirow[t]{2}{*}{16.65} & $0.48 \pm 0.02$ & \multirow[t]{2}{*}{42.10} & $0.48 \pm 0.02$ & \multirow[t]{2}{*}{45.01} & $0.48 \pm 0.02$ & \multirow[t]{2}{*}{26.68} \\
\hline & & $(27.03 \pm 0.24)^{* \#}$ & & $(29.73 \pm 0.5)^{* \#}$ & & $(29.73 \pm 0.54)^{* \#}$ & & $(29.73 \pm 0.49)^{* @ \#}$ & \\
\hline \multirow[t]{2}{*}{ (250) } & \multirow[t]{2}{*}{$0.4 \pm 0.006$} & $0.56 \pm 0.03$ & \multirow[t]{2}{*}{23.34} & $0.45 \pm 0.02$ & \multirow[t]{2}{*}{75.66} & $0.45 \pm 0.02$ & \multirow[t]{2}{*}{76.87} & $0.48 \pm 0.04$ & \multirow[t]{2}{*}{50.66} \\
\hline & & $(40 \pm 1.9)^{\#}$ & & $(12.5 \pm 0.62)^{*}$ & & $(12.5 \pm 0.23)^{*}$ & & $(20 \pm 0.29)^{*}$ \# & \\
\hline \multirow[t]{2}{*}{ (500) } & \multirow[t]{2}{*}{$0.4 \pm 0.002$} & $0.45 \pm 0.01$ & \multirow[t]{2}{*}{61.46} & $0.44 \pm 0.01$ & \multirow[t]{2}{*}{80.53} & $0.43 \pm 0.02$ & \multirow[t]{2}{*}{86.12} & $0.41 \pm 0.02$ & \multirow[t]{2}{*}{93.83} \\
\hline & & $(12.5 \pm 3.4)^{*} @$ & & $(10 \pm 0.32)^{*} @$ & & $(7.5 \pm 0.33)^{*} @$ & & $(2.5 \pm 0.16)^{*}$ & \\
\hline
\end{tabular}

Results are expressed as means \% of inhibition $\pm \mathrm{SE} ; n=8 ; P \leq 0.05 ; \%$ of increase of inflammation was measured relative to zero time of each group; \% of inhibition of inflammation measured at the same time interval for each group)

"Significant difference from saline control group at $P \leq 0.05$; ${ }^{@}$ Significant different from indomethacin group at $P \leq 0.05$; Significant different from AME 500 mg/ kg b.wt. group at $P \leq 0.05$

of paracetamol exhibited significant elevation in serum enzyme level of ALT and AST by 46.42 and 152.6\%, respectively after $24 \mathrm{~h}$ upon comparison with the control group. The elevated serum enzyme level in paracetamol group was significantly reduced by 56.87 \& $25.16 \%$ for ALT and AST, respectively after oral administration of silymarin $25 \mathrm{mg} / \mathrm{kg}$ b.wt. AME oral administration of three dose levels (125, 250 and $500 \mathrm{mg} / \mathrm{kg}$ b.wt.) showed significant reduction in elevated serum ALT by 44.36 , 55.39 and $59.3 \%$, respectively as compared with the paracetamol treated group, while the elevated level of AST was significantly reduced at dose 125 and $250 \mathrm{mg} /$ kg b.wt. by 22.74 and 27.19 , respectively upon comparison with the paracetamol treated group (Table 7).

Histopatlogical examination for liver tissue of the control rats group showed normal architecture (Fig. 4a, b). Paracetamol administration showed granularity of the cytoplasm of hepatocytes, associated with focal hepatic necrosis, inflammatory cells infiltration and portal infiltration with leucocytes (Fig. 4c-f). A liver section of rats treated with AME of $P$. racemosa (125 mg/kg b.wt.) showed diffused vacuolar degeneration of hepatocytes and mild portal infiltration with inflammatory cells (Fig. $4 \mathrm{~g}, \mathrm{~h})$. The liver section of rats treated with $250 \mathrm{mg} / \mathrm{kg}$ b.wt. revealed alteration nearly similar to those of the control. The portal area appeared normal with no inflammatory cell infiltration (Fig. $4 \mathrm{i}, \mathrm{j}$ ) while liver section of rats treated with $500 \mathrm{mg} / \mathrm{kg}$ b.wt. showed mild vacuolation of hepatocellular cytoplasm, activation of kupffer cells and increase number of binucleated hepatocytes. The Portal area showed hyperplasia of biliary epithelium and formation of newly formed bile ductuoles (Fig. 4 k).

\section{Discussion}

Structure elucidation of the isolated compounds

The chromatographic and physical properties along with NMR spectral data of compounds 1, 2, 11 and 12 were completely in agreement with the corresponding published data for gallic acid (1), methyl gallate (2), quercetin (11) and ellagic acid (12) [27-30].

Data listed in Tables 2 and 3 for compounds 3-6 matched with the available literature for quercetin 3-O- $\alpha$-L-arabinofuranoside (avicularin, 3), quercetin 3-O- $\beta-D$-arbinopyranoside (4), quercetin 3-O- $\beta$-D-glucopyranoside (5) and

Table 4 Antinociceptive activity of $P$. racemosa leaves AME and aspirin on acetic acid induced writhing in mice

\begin{tabular}{|c|c|c|c|c|}
\hline Groups & Dose mg/kg b.wt. & Number of writhing / 30 min $(X \pm S . E)$ & $\%$ Inhibition of writhing & Potency \\
\hline Saline & $1 \mathrm{ml}$ & $15 \pm 1.14$ & - & \\
\hline Aspirin & 200 & $5 \pm 0.44^{*}$ & 66.66 & 1 \\
\hline \multirow[t]{3}{*}{ AME } & 175 & $12.2 \pm 0.58^{* @}$ & 18.66 & 0.28 \\
\hline & 350 & $9 \pm 0.31^{* @ \# ~}$ & 40 & 0.6 \\
\hline & 700 & $7.6 \pm 0.4^{* @ \#}$ & 49.33 & 0.74 \\
\hline
\end{tabular}

Data represent the mean value \pm S.E; $n=5$

"Significant different from saline control group at $P \leq 0.05 ;{ }^{@}$ Significant different from aspirin group a $P \leq 0.05$, Significant difference from the extract 175 mg/kg b.wt. group a $P \leq 0.05$; Potency was calculated as regard the percentage change of the aspirin 
Table 5 Antinociceptive activity of $P$. racemosa leaves AME and tramadol on thermal pain in mice

\begin{tabular}{|c|c|c|c|c|c|c|c|}
\hline \multirow{2}{*}{$\begin{array}{l}\text { Group Dose } \\
\text { (mg/kg b.wt.) }\end{array}$} & \multirow{2}{*}{$\begin{array}{l}\text { basal } \\
\text { Latency (s) }\end{array}$} & \multicolumn{3}{|l|}{$1 \mathrm{~h}$} & \multicolumn{3}{|l|}{$2 \mathrm{~h}$} \\
\hline & & Latency (s) & Change \% & Potency & Latency(s) & Change \% & Potency \\
\hline Saline & $23.84 \pm 0.27$ & $23.07 \pm 0.6$ & 3.23 & - & $19.48 \pm 0.62$ & 18.29 & - \\
\hline Tramadol (20) & $23.21 \pm 0.81$ & $22.72 \pm 0.9$ & 2.11 & 1 & $31.48 \pm 0.4^{* @}$ & 35.63 & 1 \\
\hline \multicolumn{8}{|l|}{ AME } \\
\hline 175 & $23.01 \pm 0.7$ & $22.67 \pm 0.81$ & 1.48 & 0.7 & $24.46 \pm 0.2$ & 6.3 & 0.17 \\
\hline 350 & $23.60 \pm 0.42$ & $23.52 \pm 0.73$ & 0.34 & 0.2 & $25.82 \pm 0.7$ & 9.4 & 0.26 \\
\hline 700 & $22.90 \pm 0.5$ & $28.23 \pm 0.8^{* @}$ & 23.28 & 11 & $33.24 \pm 0.44^{* @}$ & 45.15 & 1.2 \\
\hline
\end{tabular}

Data represent the mean value \pm . S.E. $(n+6) ; n=6$

*Significantly difference from normal group at same time interval measurement $P \leq 0.05$; ${ }^{@}$ Significantly different from corresponding group at zero time interval measurement $P \leq 0.05$; Potency was calculated as regard the percentage change of the tramadol

quercetin 3-O- $\alpha$-L-rhamnopyranoside (quercetrin, 6) (27, 29-32].

Compound 7: It gave the chromatographic properties and UV data characteristic for luteolin-7-O-glycoside as well as it gave D-glucose and luteolin upon complete acid hydrolysis. Its ${ }^{1} \mathrm{H}-\mathrm{NMR}$ showed an ABX spin coupling system of three aromatic protons, H- 2'/6' $(m, \delta$ $7.45)$ and $5^{\prime}(d, \delta 6.90)$ together with singlet signal at $\delta$ 6.79 for $\mathrm{H}-3$ characteristic for the luteolin nucleus. Down field shift of H-6 $(\delta 6.45)$ and H-8 ( $\delta$ 6.77) together with doublet of $\mathrm{H}-1$ " at 5.09 revealed the presence of sugar moiety at 7-OH. Final confirmation of 7 was done through its ${ }^{13} \mathrm{C}-\mathrm{NMR}$ data which revealed the presence of 15 carbons resonance for luteolin nucleus especially for C-3 (103.64) as well as the characteristic position of $\mathrm{C}-7$ resonance (162.86 $\mathrm{ppm}$ ) together with the six carbons for glucose moiety which confirmed the location of glucose moiety at C-7 [31, 32].

Compound 8: It showed the chromatographic characters and UV data for ellagitannins [33]. On complete acid hydrolysis, ellagic and gallic acids were liberated into the organic layer while glucose was detected in the aqueous layer (CO-PC). ${ }^{1} \mathrm{H}-\mathrm{NMR}$ of 8 showed two singlets each for one proton at $\delta 6.42$ and 6.40 for $\mathrm{H}-3^{\prime \prime}$ ' and H-3"' of hexahydroxydiphenoyl (HHDP) group, together with singlet signal for two protons $\mathrm{H}-2^{\prime} / 6^{\prime}$ of galloyl moiety in the range at $6.97 \mathrm{ppm}$. The sugar moiety was confirmed as $\beta-{ }^{4} C_{1}$ glucose from the doublet signal for $\mathrm{H}-1$ at $\delta 5.70(8.7 \mathrm{~Hz})$ [33-35]. Downfield shift of $\mathrm{H}-1, \mathrm{H}-6_{\mathrm{a}}$ and $\mathrm{H}-4$ at 5.70, 4.86 and 4.60, respectively gave an evidence for attachment of galloyl moiety on $\mathrm{OH}-1$ and bi-functioned esterification of both $\mathrm{OH}-4$ and OH-6 with the HHDP moiety because of the anisotropic effect of the deshielding cone of the upper phenyl ring of HHDP on H-6a. Further confirmation of the structure was achieved via ${ }^{13} \mathrm{C}$-NMR data which revealed the downfield of C-1 (94.73) and up field of C-2 (71.61) due to the galloylation of $\mathrm{OH}-1$. Moreover, according to ${ }^{13} \mathrm{C}$-substituent additive rules, esterification of $\mathrm{OH}-6$ and $\mathrm{OH}-4$ with HHDP was confirmed from the downfield of C-4 (71.61) and C-6 (62.74) as well as upfield shift of C-3 (73.82) and C-5 (73.33) [36]. The presence of galloyl group was established from the presence of five carbon atoms characteristic for gallic acid. Moreover, the resonance of 14 carbon atoms revealed the presence of HHDP moiety. Therefore, compound $\mathbf{8}$ was identified as 1-O-galloyl-4, 6-(S)-hexahydroxydiphenoyl- $\beta$-D-glucopyranose (Strictinin).

Compound 9: It exhibited the chromatographic properties and the very broad strong UV absorption maxima $(290 \mathrm{~nm})$ characteristic for C-glycosidic ellagitannin. Moreover, it gave ellagic acid in the organic phase with unknown intermediate ellagitannin on complete acid hydrolysis in addition to the absence of glucose in the aqueous phase which gave the conformation of the C-glycosidic nature of 9 [35]. ${ }^{1} \mathrm{H}-\mathrm{NMR}$ data revealed the presence of two singlets signals one of which for two

Table 6 Effect of P. racemosa leaves AME and ranitidine on gastric mucosal injury induced by ethanol in rats

\begin{tabular}{llllll}
\hline Treated groups & Dose $\mathrm{mg} / \mathrm{kg}$ b.wt. & Number of lesions/rat & \% change & Severity of lesions/rat & \% change \\
\hline Ethanol (70\%) & $1 \mathrm{~mL}$ & $16.20 \pm 0.37$ & - & $56.8 \pm 0.97$ & - \\
Ranitidine & 50 & $2.0 \pm 0.31^{*}$ & 87.7 & $3.2 \pm 0.37^{*}$ & 94.4 \\
AME & 125 & $8.6 \pm 0.24^{* @}$ & 46.9 & $26.8 \pm 0.37^{* @}$ & 52.8 \\
& 250 & $3.4 \pm 0.24^{* @ \#}$ & 79 & $10 \pm 0.44^{* @ \#}$ & 82.4 \\
& 500 & $2.8 \pm 0.37^{* \#}$ & 82.7 & $4.8 \pm 0.37^{* \#}$ & 91.5 \\
\hline
\end{tabular}

Data is represented as means \pm S.E; $n=5$; Data were analyzed using one way analysis of variance (ANOVA) followed by Tukey Kramer multiple comparisons test; Significant at $P \leq 0.05$

"Significant difference from positive control ethanol group; ${ }^{\circledR}$ Significant different from ranitidine group; ${ }^{\#}$ Significant difference from the extract $(125 \mathrm{mg} / \mathrm{kg})$ group 


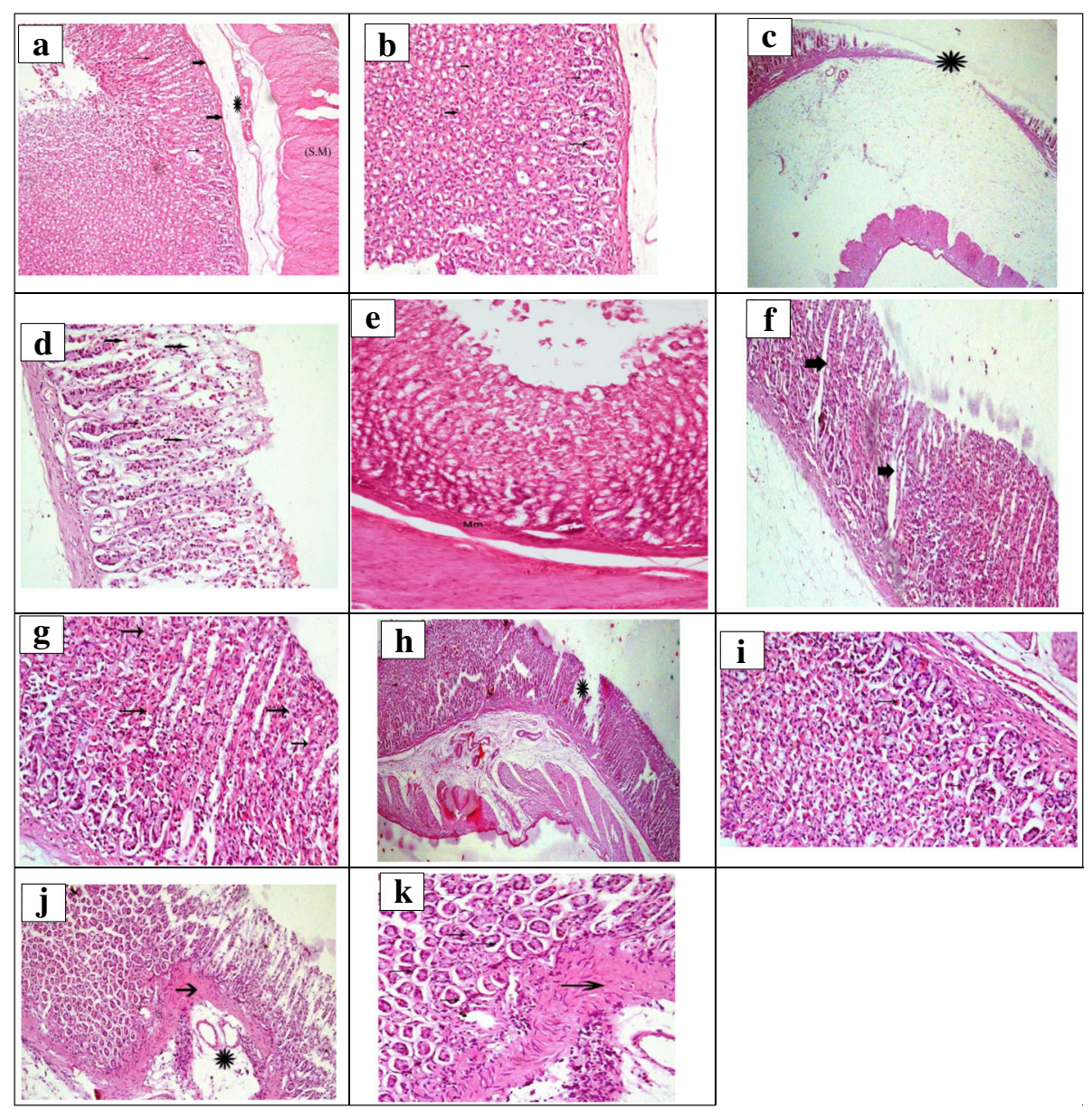

Fig. 3 Anti gastro-ulcerogenic effect of $P$. racemosa leaves AME. a A photomicrograph of gastric tissue for negative control group, showed normal stomach tissue which is divided histologically into mucosal layer formed of straight tubular glands (thin arrow) then lined by the sub mucosa (thick arrow) and the connective tissue of the sub mucosa $\left(^{*}\right)$ finally the smooth muscle layer (S.M) (H\&E X 100). b Higher magnification of (a) showed, the chief cells (thin arrow) from which mainly the glands formed with scattered parietal cells known by its eosinophilic cytoplasm (thick arrow) (H\&E X 200). c A photomicrograph of gastric tissue for positive control (ethanol group), showed ulcerated stomach tissue with loss of most of the mucosal glands $\left(^{*}\right)(H \& E$ X40). $\mathbf{d}$ Higher magnification of the (c) showed the area of ulceration with disrupted glands (thin arrow) and others are atrophied (thick arrow) (H\&E X 200). e A photomicrograph of gastric tissue for ranitidine $50 \mathrm{mg} / \mathrm{kg}$ b.wt. treated group showed slight ulcerated and distorted mucous membrane (H\&E X100). $\mathbf{f}$ A photomicrograph for of gastric tissue in rats treated with AME of $P$. racemosa leaves (125 mg/kg b.wt.) showed the ulcerated area with losing the mucosal glands (thick arrow) (H\&E X 100). g Higher magnifications of (f), showed sever destruction of the mucosal glands (thin arrow) (H\&E X 200). $\mathbf{h}$ A photomicrograph for of gastric tissue in rats treated with AME of P. racemosa leaves $(250 \mathrm{mg} / \mathrm{kg}$ ) showed the ulcerated area without healing features $(*)(H \& E X 100)$. i Higher magnification of (h), showed exaggerated parietal cells with its eosinophilic cytoplasm (thin arrow) (H\&E X 200). j A photomicrograph for of gastric tissue in rats treated with AME of $P$. racemosa leaves ( $500 \mathrm{mg} / \mathrm{kg}$ b.wt.) showed ulcerated area started to be healed $\left(^{*}\right)$ by formation of the granulation tissue (thick arrow) (H\&E X 100). $\mathbf{k}$ Higher magnification of (j). showed the granulation tissue formed of connective tissue fibers and minute blood vessels formed, beneath this tissue there are gradual revolution of the glands (thin arrow) (H\&E X 200)

proton at $\delta 6.69 \mathrm{ppm}$ and the other for one proton at $\delta$ $6.50 \mathrm{ppm}$ characteristic for a C-glycosidic flavogallonoyl group attached to $\mathrm{C}-1, \mathrm{C}-2, \mathrm{C}-3$ and $\mathrm{C}-5$ and HHDP at $\mathrm{C}-4$ and $\mathrm{C}-6$ of an open chain glucose. C-glycosidic structure of 9 was established from the $\delta \mathrm{ppm}$ and J-values of the glucose moiety, especially that of $\mathrm{H}-1$ at $\delta$ $5.51(4.0 \mathrm{~Hz})$, which confirmed the full substituted open glucose with anomeric axial $\mathrm{OH}$-group [35, 37, 38]. More conformation of the compound was done from its ${ }^{13} \mathrm{C}$-NMR data which showed 14 carbon resonances for
HHDP moiety together with 21 carbon resonance characteristic for flavogallonoyl units attached by $\mathrm{C}$-glycosidic linkage with $\mathrm{C}-1$ of glucose. The open chain glucose unit instead of hemiacetal ${ }^{4} \mathrm{C}_{1}$-pyranose was clearly proved due to the strong upfield location of $\mathrm{C}-1$ at $\delta 66.35$ in comparison to that of pyranose form $(+\approx$ 90-95 ppm). Moreover, the downfield shift of C-3' at $\delta$ $113.78(\approx+10 \mathrm{ppm})$ and upfield shift of the carbonyl carbon C-7' at $164.96(\approx-6 \mathrm{ppm})$ of the flavogallonoyl moiety gave more conformation about the $\mathrm{C}$-glycosidic 
Table 7 Effects of $P$. racemosa leaves AME and silymarin on ALT and AST in paracetamol induced hepatotoxicity in rats

\begin{tabular}{|c|c|c|c|c|c|c|c|c|c|c|}
\hline \multirow{3}{*}{$\begin{array}{l}\text { Item } \\
\text { (IU/L) }\end{array}$} & \multicolumn{10}{|c|}{ Group (dose in mg/kg b.wt.) } \\
\hline & \multirow{2}{*}{$\begin{array}{l}\text { Negative } \\
\text { Control }\end{array}$} & \multirow[t]{2}{*}{ Paracetamol640 } & \multirow[t]{2}{*}{ Silymarin 25} & \multirow{2}{*}{$\begin{array}{l}\% \text { of } \\
\text { change }\end{array}$} & \multicolumn{6}{|c|}{$80 \%$ AME of $P$. racemosa leaves } \\
\hline & & & & & 125 & $\%$ of change & 250 & $\%$ of change & 500 & $\%$ of change \\
\hline$\overline{\mathrm{ALT}}$ & $15.61 \pm 0.65^{*}$ & $46.42 \pm 1.8$ & $20.02 \pm 1.8^{*}$ & 56.87 & $25.83 \pm 2.4^{*} @$ & 44.36 & $20.71 \pm 1.58^{*}$ & 55.39 & $18.89 \pm 1.17^{*}$ & 59.3 \\
\hline AST & $94.77 \pm 7.6^{*}$ & $152.6 \pm 7.2$ & $114.2 \pm .2 .0^{*}$ & 25.16 & $117.9 \pm 2.0^{*}$ & 22.74 & $111.1 \pm 4.28^{*}$ & 27.19 & $138.6 \pm 4.7$ & 9.2 \\
\hline
\end{tabular}

Values represent the mean \pm S.E. Data is represented as means \pm S.E; $n=8$; Data were analyzed using one way analysis of variance (ANOVA) followed by Tukey Kramer multiple comparisons test. Significant at $\mathrm{P} \leq 0.05$

"Significant difference from positive control paracetamol group; ${ }^{\circledR}$ Significant difference from negative control group; Percent of change was calculated as regard to paracetamol control group

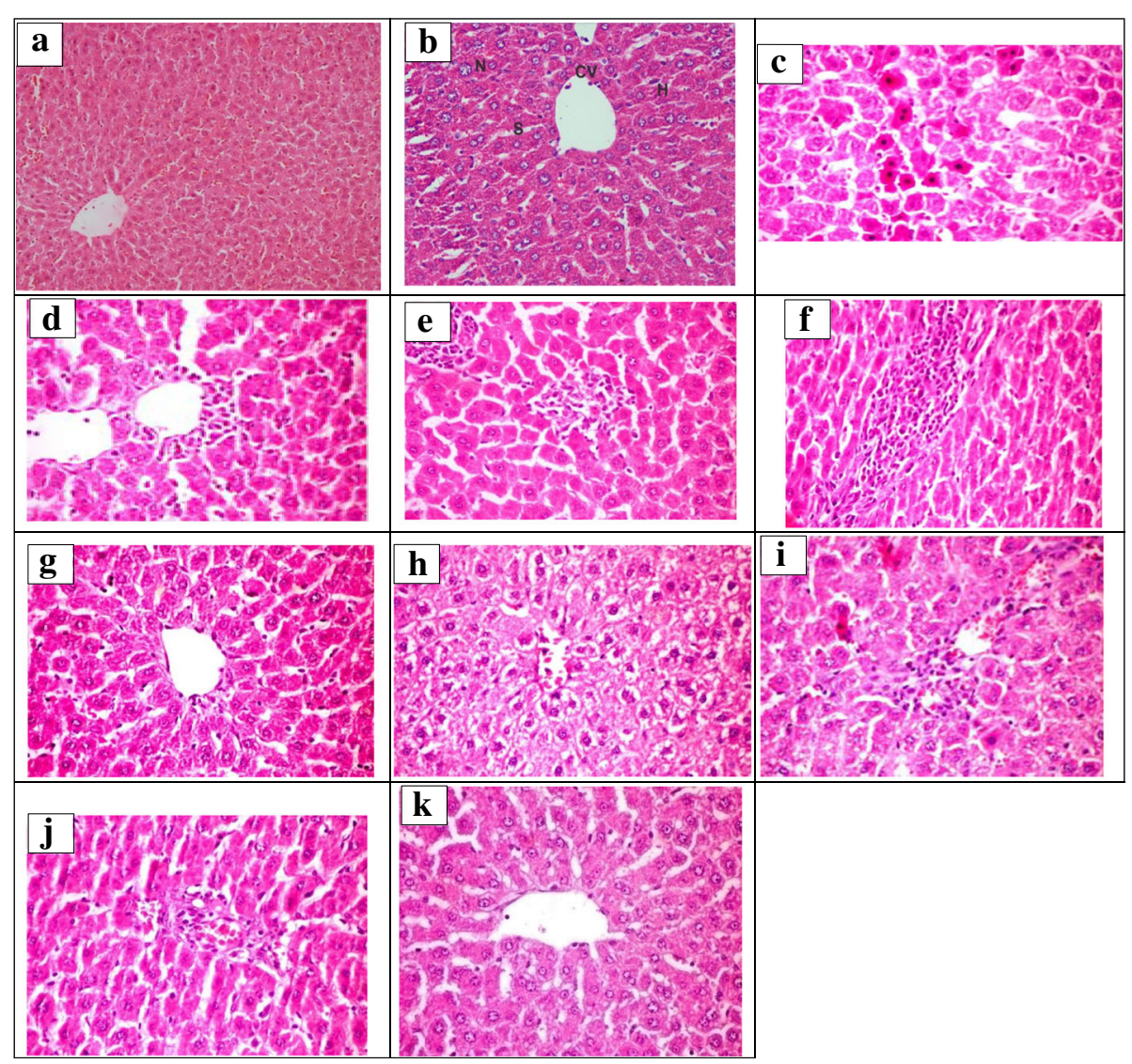

Fig. 4 Hepatoprotective activity of $P$. racemosa leaves AME. a, b A photomicrograph of a control group liver section showing normal hepatic architecture with well-arranged hepatic cords, the central vein $(C V)$ and hepatic cords of hepatocytes $(H)$ with prominent nucleus $(N)$ separated with blood sinusoids (S). No evidence of necrosis or inflammatory reaction in the portal area could be observed (H\&E X 100 \& 200). c A photomicrograph of positive control liver section showed disorganization of hepatic cords associated with sporadic cell necrosis (H\&E X 400). d, e A photomicrograph of positive control liver section showed centri lobular as well as focal area of hepatocellular necrosis infiltrated by mononuclear cells (H\&E X 400). f A photomicrograph of positive control liver section showed portal areas were intensely infiltration with inflammatory cells mostly mononuclear cells (H\&E X 400). $\mathbf{g}$, h A photomicrograph of a liver section rats treated with AME of P. racemosa leaves (125 mg/kg b.wt.) showed diffuse vacuolar degeneration of hepatocytes and mild portal infiltration with inflammatory cells (H\&E X 400). i A photomicrograph of liver section treated with AME of P. racemosa leaves $(250 \mathrm{mg} / \mathrm{kg}$ b.wt.) showed alteration nearly similar to those of the control one with focal sinusoidal dilatation and increased number of binucleated hepatocytes (H\&E X 400). j A photomicrograph of a liver section rats treated with AME of P. racemosa leaves (250 mg/kg b.wt.) showed portal area appeared normal with no inflammatory cell infiltration (H\&E.X.400). $\mathbf{k}$ A photomicrograph of a liver section rats treated with AME of P. racemosa leaves (500 mg/kg b.wt.) showed mild vacuolation of hepatocellular cytoplasm, activation of kupffer cells and increase number of binucleated hepatocytes. Portal area showed hyperplasia of biliary epithelium and formation of newly formed bile ductuoles (H\&E X 400) 
nature. The $\delta$ ppm and $\mathrm{J}_{12}$ - values of $\mathrm{H}-1$ glucose proved that the absolute configuration at the anomeric position should be of axial $\mathrm{OH}$ and equatorial $\mathrm{H}$. Additionally, UPLC-ESI-MS negative mode spectrum of $\mathbf{9}$ showed pseudo-molecular ion peaks at $\mathrm{m} / z 933$ assigned to $[\mathrm{M}-\mathrm{H}]^{-}$which matched with the reported molecular weight (934) of castalagin [39]. Accordingly, compound 9 was identified as 2,3,5-(S)-flavogalonoyl-4,6-(S)-hexahydroxydiphenoyl-D-glucose (Castalagin) [35, 37, 38].

Compound 10: It showed the chromatographic properties and UV absorption characteristic for C-glycosidic ellagitannin. $C$-glycosidic structure of $\mathbf{1 0}$ was expected from its complete acid hydrolysis which gave ellagic acid with unknown intermediate ellagitannins as well as the absence of glucose in the aqueous hydrolysate (Co-PC), [35]. Its ${ }^{1} \mathrm{H}$-NMR explained its identity as an equimolecular mixture [40]. In the aromatic region, eight singlets integrated in total to three protons, two of which for HHDP group (H-6" "/6"'") at $\delta$ ppm 6.63, 6.61, 6.62, 6.59 and the 3rd one for H-6" " at 7.32, 6.79, 6.79, 6.78 ( $1 \mathrm{H}$ in total, each s) assigned to one C-glycosidic flavogallonoyl moiety in four stereoisomers. Additionally, the repetition of some sugar resonances in the aliphatic region as $\mathrm{H}-1$ at $\delta$ ppm 4.01 and 3.98 appeared as two br s signals which supported the presence of the equimolecular isomeric mixture. Appearance of $\mathrm{H}-1$ as br s signals together with J-values of the other resolved resonances was approved with the stereo structure of this moiety in previously reported grandinin [41]. The four stereoisomers mixture for $\mathbf{1 0}$ was established from the presence of carbon resonance at $\delta$ ppm 101.15, 101.07 and 99.42, 99.34 which attributed to the hemiketal carbon resonance of the pentose moiety with carbon resonance at $47.03,46.96$ and 45.86 for C-1 due to isomerism of the pentose moiety between pyranose and furanose as well as anomeric equilibria in both conformers of sugars. The presence of pentopyranose and pentofuranose equilibrium was evidenced also from the C-4 pentose resonance as four signals at $\delta$ ppm 80.06, 79.3, 72.09 and 69.10 indicative for the four stereoisomers mixture. All the remaining resonances of ${ }^{1} \mathrm{H}$ and ${ }^{13} \mathrm{C}$ NMR spectra of 10 were assigned by comparison with previously published data on grandinin $[40,41]$. Finally, UPLC-ESI-MS negative mode spectrum of 10 showed pseudo-molecular ion peaks at $m /$ $z 1065$ assigned to $[\mathrm{M}-\mathrm{H}]^{-}$which confirmed the molecular weight of grandinin (1066) as reported before [38].

\section{Biological activities}

Based on chemical and spectroscopic evidences, the present study showed that AME of $P$. racemosa leaves contains flavonol aglycones, flavonol glycosides, flavone glycosides, phenolic acids and hydrolysable tannins, which gave gallic and ellagic acids degradation products upon hydrolysis.
AME showed significant antioxidant activities as indicated by its high DPPH scavenging percentage (89.67\%) and low $\mathrm{SC}_{50}$ value $(4.6 \mu \mathrm{g} / \mathrm{mL})$, which is mainly attributed to its constituents. Phenolic and flavonoids are responsible for a wide variety of pharmacological activities [42], which depend on their structure as antiulcer [43], analgesic anti-inflammatory [44], antioxidant [45] and hepatoprotective [46]. Their biological activities were dependent on the degree of hydroxylation, substitutions and conjugations as well as the degree of polymerization [47]. Most of the potential health benefits of flavonoids arise from their antioxidant activities that are mediated by hydroxyl groups in flavonoids which scavenge free radicals and/or by chelate metal ions [48].

Hydrolysable tannins are considered as outstanding antioxidant agents due to the presence of several gallic and hexahydroxy diphenyl groups which possess the abilities to provide protons and formation of stable free radicals which enables them to be the major active groups in the tannins molecule $[49,50]$. Moreover, the linkage between the monomers of hydrolysable tannins and phenolic hydroxyl groups as well as the esteric and the glycosidic bond also plays an important role in their antioxidants properties. The antioxidant properties of ellagitannins are responsible for their different biological activities as antiulcer [51], hepatoprotective [52] as well as anti-inflammatory [50]. It is worthy to notice that the activity can be attributed not only to phenolic compounds but also to other constituents that may participate by a role or exhibit synergistic effect as reported for other Pimenta species where the methanol extract of $P$. racemosa var. ozua leaves showed significant anti-inflammatory activity against acute edema, both when taken orally and topically [53] while its aqueous extract exhibited antinociceptive and anti-inflammatory activities [7]. Additionally, the anti-inflammatory activity of abietic acid isolated from $P$. racemosa var. grissea was proved [54].

Similarly, anti-inflammatory, analgesic, antipyretic and gastric antiulcer activities were proven for aqueous suspension of $P$. dioica (L.) in animal models where combination of anti-inflammatory and antiulcer activity represented a remarkable discovery for the ideal anti-inflammatory agent without the potential adverse effects on the gastrointestinal tract [55] which matched with our results for AME of $P$. racemosa leaves. Additionally, the antiulcerogenic and hepatoprotective activities were proved for other member of Myrtaceae by using animal models [56].

\section{Conclusion}

This paper proved the isolation and identification of phenolic constituents of AME of $P$. racemosa leaves in addition to its activities as antioxidant, analgesic anti-inflammatory, antinociceptive, gastro and hepatoprotective. This is 
hopeful for further phytochemical and biological investigations to confirm the possibility of their therapeutic effects, which may be important for the development of new natural drugs.

\section{Abbreviations}

ALT: Alanine aminotransferase; AME: Aqueous methanol extract; AST: Aspartate aminotransferase; DPPH: 2,2-Diphenyl-1-picrylhydrazyl; PI: Percentage inhibition; $\mathrm{SC}_{50}$ : The concentration required to scavenge DPPH by 50\%; UPLC-ESI-MS: Ultra performance liquid chromatographyElectrospray ionization-Mass spectrometry

\section{Acknowledgments}

The authors would like to express their deep appreciation to MSA University for providing the lab facilities and equipment for chromatographic investigation. The authors are grateful to Ass. Prof. Azza Hassan, Department of Pathology, Faculty of Veterinary Medicine, Cairo University for her unlimited help during histopathological studies.

\section{Availability of data and materials}

The data sets used and/or analyzed during the current study available from the corresponding author on reasonable request.

\section{Authors' contributions}

FAM and AAA-G helped in plant extraction, chromatographic isolation of compound and they interpreted the spectroscopic data for structure elucidation of the isolated compounds. AAA-G is the corresponding author. SE-S and $\mathrm{BI}$ conducted animal experiments for antinociceptive, anti-inflammatory, anti-ulcerogenic and hepatoprotective activities and interpreted data of biological studies. MAZ carried out the experimental work for plant extraction, isolation of compounds and helped in interpretation of results. All authors have made substantial contributions to conception and design, acquisition of data, or analysis and interpretation of data. Also, all authors have been involved in drafting the manuscript and revising it critically for important intellectual content. A final approval of the version to be published was given by all authors and they take public responsibility for appropriate portions of the content and agreed to be accountable for all aspects of the work in ensuring that questions related to the accuracy or integrity of any part of the work are appropriately investigated and resolved. All authors read and approved the final manuscript.

\section{Ethics approval}

All animal procedures were carried out according to the Ethics Committee of the National Research Centre, Cairo, Egypt (Ethics NO. 17-021) and followed the guidelines of the National Institutes of Health Guide for Care and Use of Laboratory Animals.

\section{Consent for publication}

Not applicable.

\section{Competing interests}

The authors declare that they have no competing interests.

\section{Publisher's Note}

Springer Nature remains neutral with regard to jurisdictional claims in published maps and institutional affiliations.

\section{Author details}

'Department of Pharmacognosy, Faculty of Pharmacy, Helwan University, Cairo 11795, Egypt. ${ }^{2}$ Department of Pharmacognosy, Faculty of Pharmacy, Zagazig University, Zagazig 44519, Egypt. ${ }^{3}$ Department of Pharmacology, National Research Center, Dokki, Cairo 12622, Egypt. ${ }^{4}$ Department of Pharmacognosy, Faculty of Pharmacy, Modern Sciences and Arts University, 6 October City, Egypt. ${ }^{5}$ Present Address: Department of Pharmacognosy, Faculty of Pharmacy, Modern University for Technology and Information, Cairo, Egypt.
Received: 15 April 2017 Accepted: 18 June 2018

Published online: 05 July 2018

\section{References}

1. Bailey LH. Manual of cultivated Plants. New York: The MacMillan Company; 1968.

2. Aboutabl EA, Tadros SH, El-Azzouny AMA. (2003). Composition and antimicrobial activity of volatiles from the leaf, flower and fruit of Pimenta officinalis (berg.) L. and Pimenta racemosa (mill) J.W. Moore cultivated in Egypt. Egypt Pharm J. 2003;1:45-54.

3. Alitonou GA, Noudogbessi J, Sessou P, Tonouhewa A, Avlessi F, Menut C, Sohounhloue DC. Chemical composition and biological activities of essential oils of Pimenta racemosa (mill) JW Moore from Benin. Int J Biosci. 2012;2:1-12. https://doi.org/10.4314/ijbcs.v6i4.37.

4. Duke JA, Bogenschutz-Godwin MJ, Ottesen AR. Duke's handbook of medicinal plants of Latin America. London, New York: Taylor Francis Inc, CRC Press; 2008. https://doi.org/10.1201/9781420043174.

5. Defilipps RA, Maina SL, Crepin J. Medicinal plants of the Guianas (Guyana, Surinam, French Guiana). Washington DC: Department of Botany, National Museum of Natural History, Smithsonian Institution; 2004.

6. Yousif F, Hifnawy MS, Soliman G, Boulos L, Labib T, Mahmoud S. Large-scale in vitro. Screening of egyptian native and cultivated plants for schistosomicidal activity. Pharm Biol. 2007;45(6):501-9. https://doi.org/10. 1080/13880200701389425.

7. García MD, Fernández MA, Alvarez A, Saenz MT. Antinociceptive and antiinflammatory effect of the aqueous extract from leaves of Pimenta racemosa var. ozua (Mirtaceae). J Ethnopharmacol. 2004;91 (1):69-73. https://doi.org/10. 1016/j.jep.2003.11.018.

8. Kikiuzaki H, Kawaiy HS, Nakatani N. Antioxidative phenylpropanoids from berries of Pimenta dioica. Phytochemistry. 1999;52:1307-12. https://doi.org/ 10.1016/s0031-9422(99)00406-9.

9. Kikiuzaki H, Sato A, Mayahara Y, Nakatani N. Galloylglucosides from berries of Pimenta dioica. J Nat Prod. 2000;63(6):749-52. https://doi.org/ $10.1021 / \mathrm{np} 9906121$.

10. Marzouk MS, Moharram FA, Mohamed MA, Gamal-Eldeen AM, Elsayed AA. Anticancer and antioxidant tannins from Pimenta dioica leaves. Z Naturforsch C. 2007;62(7-8):526-36. https://doi.org/10.1515/znc-2007-7-811.

11. Moharram FA, Mohamed MA, Marzouk MS, Aboutabl AE. Flavonoids and triterpenoid saponins from Pimenta dioica (Merr.) L. Nat Prod Commun. 2007;2(9):895-900. https://doi.org/10.1515/znc-2007-7-811.

12. Nayak Y, Abhilash D, Vijayanarayana K, Fernandes J. Antioxidant and hepatoprotective activity of Pimenta Dioica leaves extract. I Cell Tissue Res. 2008;8(3):1571-6.

13. Yen GC, Duh PD. Scavenging effect of methanolic extracts of peanut hulls on free radical and active oxygen species. J Agric Food Chem. 1994;42:62932. https://doi.org/10.1021/jf00039a005

14. Winter CA, Risley EA, Nuss GW. Carrageenan-induced oedema in hind paws of the rat as an assay for anti-inflammatory drugs. Proc Soc Exp Biol Med. 1962;111:544-52. https://doi.org/10.3181/00379727-111-27849.

15. Obukowicz MG, Welsch DJ, Salsgiver WJ, Berger M, Chinn KS, Duffin KL. Novel, selective delta 6 or delta 5 fatty acid desaturase inhibitors as antiinflammatory agents in mice. J Pharmacol Exp Ther. 1998;287:157-62. https://doi.org/10.1007/bf02562269.

16. Collier HDJ, Dinnin LC, Johnson CA, Schneider C. The abdominal response and its suppression by analgesic drugs in the mouse. $\mathrm{Br} \mathrm{J}$ Pharmacol Chemother. 1968;32:295-310. https://doi.org/10.1111/j.14765381.1968.tb00973.x.

17. Eddy NB, Leimbach D. Synthetic analgesics II. Dithienylbutenyl and dithienylbutilamines. J Pharmacol Exp Ther. 1953;107:385-93.

18. Wolfe AM, Kennedy LH, Na JJ, Nemzek-Hamlin JA. Efficacy of tramadol as a sole analgesic for postoperative pain in male and female mice. J Am Assoc Lab Anim Sci. 2015;54(4):411-9.

19. Robert A, Nezamis JE, Lancaster C, Hanchar AJ. Cytoprotection by prostaglandins in rats. Prevention of gastric necrosis produced by alcohol, $\mathrm{HCl}, \mathrm{NaOH}$, hypertonic $\mathrm{NaCl}$, and thermal injury. Gastroenterology. 1979;77:433-43.

20. Alvares A, Pomar F, Sevilla MA, Montero MJ. Gastric antisecretory and antiulcer activities of an ethanolic extract of Bidens pilosa $\mathrm{L}$. var. radiate Schult. J Ethnopharmacol. 1999;67:333-40. https://doi.org/10.1016/s03788741(99)00092-6. 
21. Mózsik Gy MF, Javor T. Cellular mechanism of the development of gasric mucosal damage and gasric cytoprotection induced by prostacyclin in rats. A pharmacological study. Prostaglandins Leukot Med. 1982;9(1):71-84. https://doi.org/10.1016/0262-1746(82)90074-9.

22. Drury RAB, Wallington FA. Corleton's histological technique $4^{\text {th }}$ Ed. Oxford, New York, Toronto: Oxford University press; 1980.

23. Gilani $\mathrm{AH}$, Janbaz $\mathrm{KH}$. Preventive and curative effects of Artemisia absinthium on acetaminophen and $\mathrm{CCl}_{4}$-induced hepatotoxicity. Gen Pharmacol. 1995; 26(2):309-15. https://doi.org/10.1016/0306-3623(94)00194-r.

24. Bhandari U, Shamsher A, Pillai KK. Antihepatotoxic activity of ginger ethanol extract in rats. Pharm Biol. 2003;41(1):66-71. https://doi.org/10. 1076/phbi.41.1.68.14697.

25. Sorg DA, Buckner BA. Simple method of obtaining venous blood from small laboratory animals. Proc Soc Exp Biol Med. 1964;115:1131-2. https://doi.org/ 10.3181/00379727-115-29134.

26. Semler DE, Gad SC, Chengelis CP. The rat. In: Gad SC, Chengelis CP, editors. Animal Models in Toxicology. New York: Marcel Dekker, Inc; 1992. https:// doi.org/10.1002/jat.2550130517.

27. Mabry TJ, Markham KR, Thomas MB. The systematic identification of flavonoids. New York: Springer Verlag; 1970. https://doi.org/10.1007/978-3-642-88458-0.

28. Barakat HH, Hussein SAM, Marzouk MS, Merfort I, Leinsheid MW, Nawar MAM. Polyphenolic metabolites of Epilobium hirsutum. Phytochemistry. 1997:46:935-41. https://doi.org/10.1016/s0031-9422(97)00370-1.

29. Metwally AM, Omar AA, Harraz FM, El Sohafy SM. Phytochemical investigation and antimicrobial activity of Psidium guajava L. leaves. Pharmacogn Mag. 2010; 6(23):212-8. https://doi.org/10.4103/0973-1296.66939.

30. Wan C, Yu Y, Zhou S, Tian S, Cao S. Isolation and identification of phenolic compounds from Gynura divaricata leaves. Pharmacogn Mag. 2011;7(26): 101-8. https://doi.org/10.4103/0973-1296.80666.

31. Agrawal PK, Bansal MC. Flavonoid glycosides. In: Agrawal PK, editor. Studies in Organic Chemistry 39, ${ }^{13} \mathrm{C}$ NMR of flavonoids. New York: Elsevier; 1989. https://doi.org/10.1016/b978-0-444-87449-8.50012-2.

32. Mabry TJ, Markham KR. The flavonoids. London: Chapman and Hall; 1975. https://doi.org/10.1007/978-1-4899-2909-9.

33. Gupta RK, Al-Shafi SM, Layden K, Haslam E. The metabolism of gallic acid and hexahydroxydiphenic acid in plants. Biochem Soc Trans. 1982;1:252534. https://doi.org/10.1039/p19820002525.

34. Okuda Y, Yoshida T, Hatano T, Yazaki K, Ashida M. Ellagitannins of the Casuarinaceae, Stachyuraceae and Myrtaceae. Phytochemistry. 1980;21(12): 2871-4. https://doi.org/10.1016/0031-9422(80)85058-8.

35. Okuda T, Yoshida T, Ashida M, Yazaki KJ. Tannins of casuarina and stachyurus species. Part 1. Structures of pendunculagin, casuarictin, strictinin, casuarinin, casuariin, and stachyurin. Chem Soc Perkin Trans. 1983; 1:1765-72. https://doi.org/10.1002/chin.198348342.

36. Okuda T, Yoshida T, Hatano TJ. New methods of analyzing tannins. Nat Prod. 1989;52:1-31. https://doi.org/10.1021/np50061a001.

37. Vivas N, Laguerre M, Glories Y, Bourgeois G, Vitry C. Structure simulation of two ellagitannins from Quercus robur L. Phytochemistry. 1995;39:1193-9. https://doi.org/10.1016/0031-9422(95)00148-z.

38. Moharram F, Marzouk MS, El-Toumy SAA, Ahmed AAE, Aboutabl EA Polyphenols of Melaleuca quinquenervia leaves - pharmacological studies of grandinin. Phytother Res. 2003;17:767-73. https://doi.org/10.1002/ptr.1214.

39. Rasines-Perea Z, Jacquet R, Jourdes M. C-glucosidic ellagitannin concentrations variability during the years in Syrah wines from Languedoc vineyard. BIO Web of Conferences. 2016;7:1-4. https://doi.org/10.1051/ bioconf/2016070200, https://doi.org/10.1016/0262-1746(83)90032-x.

40. Nonaka G, Ishimaru K, Azuma R, Ishimatsu M, Nishioka I. Tannins and related compounds, LXXXV. Structures of novel C-glycosidic ellagitannins, grandinin and pterocarinins a and B. Chem Pharm Bull. 1989;37:2071-7. https://doi. org/10.1248/cpb.37.2071.

41. Herv- Du penhoat C, Michon V, Peng S, Viriot C, Scalbert A, Gage D. Structural elucidation of new dimeric ellagitannins from Quercus robur L. Roburins A-E. Chem Soc Perkin Trans. 1991;1:1653-60. https://doi.org/10.1039/p19910001653.

42. Kumar S, Pandey A. Chemistry and biological activities of flavonoids: an overview. Scientific World J. 2013;2013:1-16. https://doi.org/10.1155/2013/ 162750 .

43. De Lira Mota KS, Dias GEN, Pinto MEF, Luiz-Ferreira A, Souza-Brito ARM, Hiruma-Lima CA. Flavonoids with gastroprotective activity. Molecules. 2009: 14:979-1012. https://doi.org/10.3390/molecules14030979.
44. Borgi W, Recio MC, Rios JL, Chouchane N. Anti-inflammatory and analgesic activities of flavonoid and saponin fractions from Zizyphus lotus (L.) lam. S Afr J Bot. 2008;74(2):320-4. https://doi.org/10.1016/j.sajb.2008.01.009.

45. Ghasemzadeh A, Ghasemzadeh N. Flavonoids and phenolic acids: role and biochemical activity in plants and human. J Med Plant Res. 2011;5(31):6697703. https://doi.org/10.5897/jmpr11.1404.

46. Agrawal AD. Pharmacological activities of flavonoids: a review. Int J Pharm Sci Nanotech. 2011:4(2):1394-8.

47. Kelly EH, Anthony RT, Dennis JB. Flavonoid antioxidants: chemistry, metabolism and structure-activity relationships. J Nutr Biochem. 2002;13(10): 572-84. https://doi.org/10.1016/s0955-2863(02)00208-5.

48. Kumar S, Mishra A, Pandey AK. Antioxidant mediated protective effect of Parthenium hysterophorus against oxidative damage using in vitro models. BMC Complement Altern Med. 2013;13:120. https://doi.org/10.1186/1472-6882-13-120.

49. Sener-Muratog G, Paskalog K, Arbak S, Hurdag C, Dulger G. Protective effect of famotidine, omeprazole, and melatonin against acetylsalicylic acidinduced gastric damage in rats. Dig Dis Sci. 2001;46(2):318-30. https://doi. org/10.1007/s10620-005-2869-1

50. Shu MH, Appleton D, Zandi K, Abu Bakar S. Anti-inflammatory, gastroprotective and anti-ulcerogenic effects of red algae Gracilaria changii (Gracilariales, Rhodophyta) extract. BMC Complement Altern Med. 2013;13: 61. https://doi.org/10.1186/1472-6882-13-61.

51. Wang Z, Hasegawa J, Wang X, Matsuda A, Tokuda T, Miura N, Watanabe T. Protective effects of Ginger against aspirin-induced gastric ulcers in rats. Yonago Acta med. 2011;54:11-9.

52. Moharram FA, Marzouk MS, El-Dib RA, El-Shenawy SM, Abdel-Rahman RF, Ibrahim RR. Hepatoprotective, gastroprotective, antioxidant activity and phenolic constituents of Quercus robur leaves. J Pharm Sci \& Res. 2015;7(11):1055-65

53. Fernández MA, Alvarez A, MD G'a, Sáenz MT. Anti-inflammatory effect of Pimenta racemosa var. ozua and isolation of the triterpene lupeol. II Farmaco. 2001;56:335-8. https://doi.org/10.1016/s0014-827x(01)01080-1.

54. Fernández MA, Tornos MP, MD G'a, de las Heras B, Villar AM, Sáenz MT. Anti-inflammatory activity of abietic acid, a diterpene isolated from Pimenta racemosa var. grissea. J Pharm Pharmacol. 2001;53(6):867-72. https://doi.org/ 10.1211/0022357011776027.

55. Al-Rehaily AJ, Al-Said MS, Al-Yahya MA, Mossa JS, Rafatullah S. Ethnopharmacological studies on allspice (Pimenta dioica) in laboratory animals. Pharm Biol. 2002;40(3):200-5. https://doi.org/10.1076/phbi.40.3.200.5829.

56. El-Shenawy SM, Marzouk MS, El-Dib RA, Abo Elyazed HE, Shaffie NM, Moharram FA. Polyplenols and biological activities of Feijoa sellowiana leaves and twigs. Rev Latinoamer Quím. 2008;36(3):103-20. https://doi.org/ $10.1055 / s-2007-98683$

\section{Ready to submit your research? Choose BMC and benefit from:}

- fast, convenient online submission

- thorough peer review by experienced researchers in your field

- rapid publication on acceptance

- support for research data, including large and complex data types

- gold Open Access which fosters wider collaboration and increased citations

- maximum visibility for your research: over $100 \mathrm{M}$ website views per year

At BMC, research is always in progress.

Learn more biomedcentral.com/submissions 